\title{
Unirational threefolds with no universal codimension 2 cycle
}

\author{
Claire Voisin \\ CNRS and École Polytechnique
}

\begin{abstract}
We prove that the general quartic double solid with $k \leq 7$ nodes does not admit a Chow theoretic decomposition of the diagonal, (or equivalently has a nontrivial universal $\mathrm{CH}_{0}$ group,) and the same holds if we replace in this statement "Chow theoretic" by "cohomological". In particular, it is not stably rational. We also deduce that the general quartic double solid with seven nodes does not admit a universal codimension 2 cycle parameterized by its intermediate Jacobian, and even does not admit a parametrization with rationally connected fibers of its Jacobian by a family of 1-cycles. This finally implies that its third unramified cohomology group is not universally trivial.
\end{abstract}

\section{Introduction}

Let $X$ be a smooth connected complex projective variety. If $\mathrm{CH}_{0}(X)=\mathbb{Z}$ (or equivalently the subgroup $\mathrm{CH}_{0}(X)_{0} \subset \mathrm{CH}_{0}(X)$ of 0 -cycles of degree 0 is 0 ), we have the Bloch-Srinivas decomposition of the diagonal (see [5]) which says that for some integer $N \neq 0$,

$$
N \Delta_{X}=Z_{1}+Z_{2} \text { in } \mathrm{CH}^{n}(X \times X), n=\operatorname{dim} X,
$$

where $Z_{2}=N(X \times x)$ for some point $x \in X(\mathbb{C})$ and $Z_{1}$ is supported on $D \times X$, for some proper closed algebraic subset $D \varsubsetneqq X$. Note that conversely, if we have a decomposition as in (10), then $\mathrm{CH}_{0}(X)=\mathbb{Z}$ because the left hand side acts as $N$ times the identity on $\mathrm{CH}_{0}(X)_{0}$ while the right hand side acts as 0 on $\mathrm{CH}_{0}(X)_{0}$, so that $N \mathrm{CH}_{0}(X)_{0}=0$ and finally $\mathrm{CH}_{0}(X)_{0}=0$ by Roitman's theorem [25] on torsion.

Denoting $K=\mathbb{C}(X)$, (1) is equivalent by the localization exact sequence to the fact that the diagonal point $\delta_{K}$ of $X_{K}$, that is, the restriction to $\operatorname{Spec}(K) \times X$ of $\Delta_{X}$, satisfies

$$
N \delta_{K}=N x_{K} \text { in } \mathrm{CH}^{n}\left(X_{K}\right)=\mathrm{CH}_{0}\left(X_{K}\right) .
$$

When there exists a decomposition as in (10) with $N=1$, we will say that $X$ admits a Chow theoretic decomposition of the diagonal. As explained in [2, Lemma 1.3], this is equivalent to saying that $\mathrm{CH}_{0}(X)$ is universally trivial, in the sense that for any field $L$ containing $\mathbb{C}$, $\mathrm{CH}_{0}\left(X_{L}\right)_{0}=0$.

By taking cohomology classes in (1), one gets as well a decomposition

$$
N\left[\Delta_{X}\right]=\left[Z_{1}\right]+\left[Z_{2}\right] \text { in } \mathrm{H}^{2 n}(X \times X, \mathbb{Z}), n=\operatorname{dim} X,
$$

where $Z_{1}, Z_{2}, D$ are as above, from which Bloch and Srinivas 5 deduce a number of interesting consequences. When there exists a decomposition as in (2) with $N=1$, that is

$$
\left[\Delta_{X}\right]=\left[Z_{1}\right]+[X \times x] \text { in } H^{2 n}(X \times X, \mathbb{Z}),
$$


where $Z_{1}$ is supported on $D \times X$, for some proper closed algebraic subset $D \subset X$, we will say that $X$ admits a cohomological decomposition of the diagonal. We started the study of this property in [28, mainly in the case of rationally connected threefolds.

In either of its forms (Chow-theoretic or cohomological), the existence of a decomposition of the diagonal is an important criterion for rationality, as noticed in 2, 28. This property is in fact invariant under stable birational equivalence, where we say that $X$ and $Y$ are stably birational if $X \times \mathbb{P}^{r} \stackrel{\text { birat }}{\cong} Y \times \mathbb{P}^{s}$ for some integers $r$ and $s$. As this property is obviously satisfied by projective space, an $X$ not satisfying this criterion is not stably rational.

We compare these two properties (the existence of a Chow-theoretic and cohomological decompositions of the diagonal) in [29, showing in particular that they are equivalent for cubic fourfolds and odd dimensional smooth cubic hypersurfaces.

It is a basic fact, proved in 28, that a smooth complex projective variety $X$ with nontrivial Artin-Mumford invariant (the torsion subgroup of $H^{3}(X, \mathbb{Z})$ ) does not admit a cohomological decomposition (and a fortiori a Chow-theoretic decomposition) of the diagonal. This is the starting point of this paper and combined with a degeneration (or specialization) argument (see Theorem 1.1), this will provide us with simple examples of smooth projective unirational threefolds with trivial Artin-Mumford invariant which do not admit a decomposition of the diagonal, hence are not stably rational. Note that these varieties also have trivial higher degree unramified cohomology groups by [12]

Let us now discuss a little more precisely the results in this paper, distinguishing the Chow-theoretic and cohomological cases.

\subsection{Chow-theoretic decomposition of the diagonal and application to stable rationality}

The first examples of unirational threefolds with nontrivial torsion in their degree 3 integral Betti cohomology were constructed by Artin and Mumford 11. These varieties are desingularizations of certain special 10-nodal quartic double solids (that is, double covers $Y \rightarrow \mathbb{P}^{3}$ ramified along a quartic surface with 10 nodes in special position). The examples we consider are smooth quartic double solids, for which the quartic surface is smooth, or desingularizations of $k$-nodal quartic double solids, for which the quartic surface has $k$ ordinary quadratic singularities, but unlike the Artin-Mumford examples, the nodes of the quartic surface will be in general position. They are unirational (see [3]), a fact which in the nodal case is immediate to prove by considering the preimages in $Y$ of lines in $\mathbb{P}^{3}$ passing through the node : these curves are rational and the family of these curves endows $Y$ with a conic bundle structure, which furthermore contains a multisection which is a rational surface, giving unirationality. If the quartic surface has at most seven nodes in general position, then $X$ has no torsion in its integral cohomology. We will prove by a degeneration argument (cf. Theorem 1.1,(i)) the following result.

Theorem 0.1. (Cf. Theorem [1.6) Let $X$ be the desingularization of a very general quartic double solid with at most seven nodes. Then $X$ does not admit an integral Chow-theoretic decomposition of the diagonal, hence it is not stably rational.

Remark $\mathbf{0 . 2}$. The family of 6 or 7 -nodal quartic double solids, or equivalently 6 or 7 -nodal quartic surfaces in $\mathbb{P}^{3}$, is not irreducible (see [15]). Observe however that there is an unique irreducible component $M$ of the space $M^{\prime}$ of nodal quartic surfaces in $\mathbb{P}^{3}$ with $k \leq 7$ nodes dominating $\left(\mathbb{P}^{3}\right)^{(k)}$ by the map $\phi: M \rightarrow\left(\mathbb{P}^{3}\right)^{(k)}$ which to a $k$-nodal quartic associates its set of nodes. This is what we mean in the Theorem above by "general quartic double solid $X$ with $k \leq 7$ nodes". Indeed, given a set $z$ of $k \leq 7$ points in general position, the set $M_{z}$ of quartics which are singular along $z$ is a projective space. One easily checks that a generic point in this projective space parameterizes a nodal quartic surface with exactly $k$ nodes. Thus if $U$ is the Zariski open set of $\left(\mathbb{P}^{3}\right)^{(k)}$ consisting of points $z$ such that the dimension of $M_{z}$ is minimal (in fact it is easily proved that this dimension is the expected dimension $34-4 k$ ), one finds that $\phi^{-1}(U)$ is a Zariski open set in a projective bundle over $U$, hence is 
irreducible. In fact, we also get results for quartic double solids with 8 or 9 nodes, but their parameter spaces are reducible and we do not know to which component of their parameter spaces our results apply.

The case of seven nodes is particularly interesting, because the intermediate Jacobian of $X$ in this case is of dimension 3 , hence is as a principally polarized abelian variety isomorphic to the Jacobian of a curve. Thus the Clemens-Griffiths criterion for rationality is satisfied in this case, and as a consequence, even the irrationality of the very general 7-nodal double solid proved above was unknown. In the case of $k \leq 6$, the irrationality of the very general $k$-nodal quartic double solid was already known using the Clemens-Griffiths criterion, (this is proved [31] for all smooth quartic double solids and we refer to [7] for the nodal case,) but its stable irrationality was unknown.

Remark 0.3. De Fernex and Fusi 13 prove that in dimension 3, rationality is stable under specialization to nodal fibers. Thus, if rationality instead of stable rationality is considered, the above statement concerning the irrationality of the very general $k$-nodal double solid with $k \leq 7$ could be obtained by applying their argument. Note however that our degeneration theorem (Theorem 1.1) works in any dimension, while theirs works only in dimension 3.

\subsection{Cohomological decomposition of the diagonal and cycle-theoretic applications}

We wish to describe in more detail in this paragraph which kind of information can be extracted from the non-existence of a cohomological decomposition of the diagonal, as this provides more explicit obstructions to stable rationality. A general reason why it should be more restrictive than the rational decomposition (2) is the following : If the positive degree cohomology $H^{*>0}(X, \mathbb{Q})$ has geometric coniveau $\geq 1$, that is, vanishes on the complement of a divisor $D \subset X$, a property which is implied by the decomposition (2), the same is true for the positive degree integral cohomology $H^{*>0}(X, \mathbb{Z})$ (possibly for a different divisor $\left.D^{\prime}\right)$. Indeed, as noted in [12, the Bloch-Ogus sheaves $\mathcal{H}^{i}(\mathbb{Z})$ on $X_{Z a r}$ associated to the presheaves $U \mapsto H^{i}(U, \mathbb{Z})$ have no $\mathbb{Z}$-torsion, as a consequence of the Bloch-Kato conjecture proved by Voevodsky [26]. It follows from this that the class $\left[\Delta_{X}\right]-[X \times x]$ vanishes on the Zariski open set $U \times X$ of $X$, where $U:=X \backslash D^{\prime}$. This shows that assuming (22), one gets a decomposition

$$
\left[\Delta_{X}\right]=\alpha+[X \times x] \text { in } H^{2 n}(X \times X, \mathbb{Z})
$$

where $\alpha$ is an integral cohomology class supported on $D^{\prime} \times X$. The decomposition (3) is a much stronger statement since it asks that the class $\alpha$ is the class of an algebraic cycle supported on $D^{\prime}$.

The following example on the other hand shows that in the absence of torsion in cohomology, we need some transcendental cohomology in order to prove the non-existence of an integral cohomological decomposition of the diagonal :

Example 0.4. If $X$ is a smooth projective variety such that $H^{*}(X, \mathbb{Z})$ has no torsion and is algebraic, that is, is generated by classes of algebraic subvarieties, then $X$ admits an integral cohomological decomposition of the diagonal. This follows from the Künneth decomposition which expresses the Betti cohomology class of the diagonal as a $\mathbb{Z}$-linear combination of classes $\operatorname{pr}_{1}^{*} \alpha \smile p r_{2}^{*} \beta$, and from the fact that all cohomology classes on $X$ are algebraic.

In the presence of non-trivial odd degree cohomology (necessarily of degree $\geq 3$ if we already have a rational decomposition as in (2)), the integral decomposition (3) becomes restrictive, even when the integral cohomology is torsion free, as shows our theorem 0.9 . The obstruction we find is a sort of secondary obstruction which we describe now. Recall that if $H^{3}\left(X, \mathcal{O}_{X}\right)=0$, the intermediate Jacobian of $X$, defined as the complex torus

$$
J^{3}(X)=H^{3}(X, \mathbb{C}) /\left(H^{3}(X, \mathbb{Z}) \oplus F^{2} H^{3}(X, \mathbb{C})\right),
$$


is an abelian variety. Furthermore the generalized Hodge conjecture [17] predicts that the Griffiths Abel-Jacobi map

$$
A J_{X}: \mathrm{CH}^{2}(X)_{h o m} \rightarrow J^{3}(X)
$$

is surjective. When $\mathrm{CH}_{0}(X)=\mathbb{Z}$, this is proved by Bloch and Srinivas [5] who even prove under this assumption the much stronger result that $A J_{X}: \mathrm{CH}^{2}(X)_{h o m} \rightarrow J^{3}(X)$ is an isomorphism (see also [23]). The situation is then similar to the case of divisors, where we have the Abel-Jacobi isomorphism $A J_{X}: \operatorname{Pic}^{0}(X) \cong J^{1}(X)$ and where it is well-known that there exists a universal (or Poincaré) divisor $\mathcal{P}$ on $J^{1}(X) \times X$ with the property that the morphism $\phi_{\mathcal{P}}: J^{1}(X) \rightarrow J^{1}(X)$, which to $l \in J^{1}(X)$ associates $A J_{X}\left(\mathcal{D}_{l}\right)$, is the identity. Our second main result in this paper is that, from this last viewpoint, codimension $\geq 2$ cycles actually behave differently than divisors, and as we will see, this is related to the cohomological decomposition of the diagonal. Let us make the following definition:

Definition 0.5. Let $X$ be a smooth projective variety such that $A J_{X}: \mathrm{CH}^{2}(X)_{h o m} \rightarrow J^{3}(X)$ is an isomorphism (note that $J^{3}(X)$ is then automatically an abelian variety). We will say that $X$ admits a universal codimension 2 cycle if there exists a codimension 2 cycle $Z \in \mathrm{CH}^{2}\left(J^{3}(X) \times X\right)$ such that $Z_{\mid a \times X}$ is homologous to 0 for $a \in J^{3}(X)$ and the morphism induced by the Abel-Jacobi map

$$
\Phi_{Z}: J^{3}(X) \rightarrow J^{3}(X), a \mapsto A J_{X}\left(Z_{a}\right),
$$

is the identity of $J^{3}(X)$.

Remark 0.6. Note that under the same assumption $H^{3}\left(X, \mathcal{O}_{X}\right)=0$, there is an integral Hodge class $\alpha$ of degree 4 on $J^{3}(X) \times X$ which is determined up to torsion by the property that

$$
\alpha_{*}: H_{1}\left(J^{3}(X), \mathbb{Z}\right) \rightarrow H^{3}(X, \mathbb{Z}) / \text { torsion }
$$

is the canonical isomorphism given by the definition (4) of $J^{3}(X)$ as a complex torus and by the fact that $\alpha$ is of type $(1,3)$ in the Künneth decomposition of $H^{4}\left(J^{3}(X) \times X, \mathbb{Z}\right)$.

The rational cohomological decomposition of the diagonal (2) implies that $N \alpha$ (modulo torsion) is algebraic (see Lemma 2.5 and [5]). So for a smooth complex projective variety $X$ with $\mathrm{CH}_{0}(X)=\mathbb{Z}$, saying that $X$ admits a universal codimension 2 cycle is equivalent to saying that this degree 4 integral Hodge class on $J^{3}(X) \times X$, which is known to be $\mathbb{Q}$-algebraic, is actually algebraic (modulo the torsion of $H^{4}\left(J^{3}(X) \times X, \mathbb{Z}\right)$ ).

We proved in 28, that if $X$ has dimension 3, the integral cohomological decomposition (3) has the following consequences (consequence 1 is due to Bloch and Srinivas [5] and uses only the rational cohomological decomposition (2) ):

Theorem 0.7. Assume a smooth projective 3-fold has an integral cohomological decomposition of the diagonal. Then

1. $H^{i}\left(X, \mathcal{O}_{X}\right)=0$ for $i>0$.

2. $H^{*}(X, \mathbb{Z})$ has no torsion.

3. The even degree integral cohomology of $X$ consists of classes of algebraic cycles.

4. There exists a universal codimension 2 cycle $Z \in \mathrm{CH}^{2}\left(J^{3}(X) \times X\right)$.

Conversely, if 1 to 4 hold and furthermore the following property holds:

5. $J^{3}(X)$ has a 1-cycle $z \in \mathrm{CH}^{g-1}\left(J^{3}(X)\right)$ whose cohomology class $[z] \in H^{2 g-2}\left(J^{3}(X), \mathbb{Z}\right)$ is the minimal class $\frac{\theta^{g-1}}{(g-1) !}$, where $g:=\operatorname{dim} J^{3}(X)$, and $\theta \in H^{2}\left(J^{3}(X), \mathbb{Z}\right)$ is the class of the natural principal polarization on $J^{3}(X)$,

then $X$ admits an integral cohomological decomposition of the diagonal. 
Remark 0.8. More recently, we proved in [29] that Property 5 above is also implied by the existence of a cohomological decomposition of the diagonal, but we will not need this result here.

If we consider a rationally connected threefold $X$, 1 and 3 are always satisfied 10 is standard and 3 is proved in [27). The Artin-Mumford example [1] shows that 2 does not always hold even for unirational threefolds and this provides by the theorem above an obstruction to the existence of an integral cohomological decomposition of the diagonal.

Property 4 is much harder to analyze and it is still unknown if it is satisfied for the general cubic threefold (this result is claimed in 32 but the proof is incorrect, as there is a missing term in the formula used for $\operatorname{ch}\left(\mathcal{O}_{X}(1)\right)$ in the proof of Theorem 2.3 of loc. cit.). We prove in [29] that it is satisfied by "many" smooth cubic threefolds, (more precisely, there is a countable union of subvarieties of codimension $\leq 3$ in the moduli space of cubic threefolds parameterizing cubic threefolds satisfying property 4 . One of our main results in this paper is the fact that Property 4 can fail for some unirational threefolds. In fact, we will exhibit unirational threefolds $X$ for which property 2 (the Artin-Mumford criterion) and property 5 of Theorem 0.7 are satisfied, but not admitting an integral cohomological decomposition of the diagonal. Such a threefold $X$ does not admit a universal codimension 2 cycle by Theorem 0.7

Theorem 0.9. (Cf. Theorem 1.6 and Theorem 1.10) (i) Let $X$ be the desingularization of a very general quartic double solid with at most seven nodes. Then $X$ does not admit an integral cohomological decomposition of the diagonal.

(ii) If $X$ is the desingularization of a very general quartic double solid with exactly seven nodes, then $X$ does not admit a universal codimension 2 cycle $Z \in \mathrm{CH}^{2}\left(J^{3}(X) \times X\right)$.

Concerning property 5 , that is, the question whether $J^{3}(X)$ has a 1-cycle in the minimal class $\frac{\theta^{g-1}}{(g-1) !}$, this is a very classical completely open question for most rationally connected threefolds (in particular the cubic threefold, for which we prove in 29 that a positive answer to this question is equivalent to the fact that the cubic threefold has universally trivial $\mathrm{CH}_{0}$ group) but also for very general principally polarized abelian varieties of dimension $\geq 4$. The Clemens-Griffiths criterion for rationality [9] states that if a smooth projective threefold is rational, its intermediate Jacobian is isomorphic as a principally polarized abelian variety to a product of Jacobians of smooth curves. By the Matsusaka characterization of products of Jacobians 22, another way to state the Clemens-Griffiths criterion is to say that there exists an effective 1-cycle in $J^{3}(X)$ (that is, a combination with positive coefficients of curves in $\left.J^{3}(X)\right)$ whose cohomology class is the minimal class $\frac{\theta^{g-1}}{(g-1) !}$. This condition is much more restrictive geometrically than Property 5 above. In particular cases, it can be solved negatively for a general $X$ by a dimension count for the number of parameters for Jacobians of curves, or for a specific $X$ by the precise study of the geometry of the Theta divisor. This cannot be done with the question of the existence of a 1-cycle in the minimal class that is not necessarily effective. We will not be concerned by this problem however, since in the examples we will analyze closely, namely the 7 -nodal quartic double solids, we will have $\operatorname{dim} J^{3}(X)=3$ so $J^{3}(X)$ will automatically satisfy the Clemens-Griffiths criterion. Together with the triviality of the Artin-Mumford invariant, this allows us to entirely focus on property 4 , which by Theorem 0.7 above is the only obstruction to the existence of a cohomological decomposition of the diagonal.

Our next result (Theorem 0.10) will relate the non-existence of a universal codimension 2 cycle to the vanishing of the universal third unramified cohomology with torsion coefficients introduced in 2. . Unramified cohomology with torsion coefficients has been used by ColliotThélène and Ojanguren [10] as a powerful tool to detect irrationality (see also [24]). In the paper [2, the authors introduce the notion of "universal triviality of the third unramified cohomology group of $X$ ". We just sketch here the idea and refer to 2 for more details. The universal triviality of the third unramified cohomology group of $X$ with torsion coefficients is equivalent to the fact that for any smooth quasi-projective variety $U$ and any class $\alpha \in$ 
$H_{n r}^{3}(U \times X, \mathbb{Q} / \mathbb{Z})$, there is a Zariski dense open set $U^{\prime} \subset U$ such that $\alpha_{\mid U^{\prime} \times X}$ is the pull-back of a class $\beta \in H^{3}\left(U^{\prime}, \mathbb{Q} / \mathbb{Z}\right)$. In the paper $[2$, the notion is more elegantly formulated since the authors can use the étale cohomology of the variety $X_{K}$ where $K$ is the function field of $U$, but in the context of Betti cohomology, we have to formulate it by taking the direct limit over Zariski open sets. In any case, the notion is obviously particularly interesting for those varieties with vanishing third unramified cohomology group with torsion coefficients, as it is the case for rationally connected threefolds (see [12]).

Theorem 0.10. (Cf. Theorem 2.1) Let $X$ be a smooth complex projective variety of dimension $n$ with $\mathrm{CH}_{0}(X)=\mathbb{Z}$. Assume

1. $H^{*}(X, \mathbb{Z})$ has no torsion and the Künneth components of the diagonal are algebraic.

2. The group $H_{n r}^{3}(X, \mathbb{Q} / \mathbb{Z})$ is trivial (or equivalently by [12], the integral Hodge classes of degree 4 on $X$ are algebraic).

Then the degree 3 unramified cohomology of $X$ with torsion coefficients is universally trivial if and only if there is a universal codimension 2 cycle $Z \in \mathrm{CH}^{2}\left(J^{3}(X) \times X\right)$.

These hypotheses apply to any rationally connected threefold with no torsion in $H^{3}$ (see Corollary [2.3), and also to cubic fourfolds, proving in particular the universal triviality of the third unramified cohomology group with torsion coefficients of any smooth cubic fourfold (see Example 2.2).

Corollary 0.11. If $X$ is as in Theorem 0.9, (ii), the universal third unramified cohomology group of $X$ with coefficients in $\mathbb{Q} / \mathbb{Z}$ is not universally trivial.

\subsection{A geometric application}

We conclude this paper with a result of a more geometric nature. It concerns the following weaker version $\left(^{*}\right)$ of property 4 of Theorem 0.7 . originating in work of de Jong and Starr (see [14), which is very natural if one thinks of the geometry of the Abel map for 0-cycles on curves:

(*) There exist a smooth projective variety $B$ and a codimension 2 cycle $Z \in \mathrm{CH}^{2}(B \times X)$ inducing a surjective map

$$
\Phi_{Z}: B \rightarrow J^{3}(X), b \mapsto A J_{X}\left(Z_{b}\right)
$$

with rationally connected general fibers.

Property $(*)$ is satisfied by cubic threefolds (see [21]). In [28, we observed that $\left(^{*}\right.$ ) has already very nice consequences on the integral Hodge conjecture (for instance, if $X$ is rationally connected and $H^{3}(X, \mathbb{Z})$ has no torsion, property $\left(^{*}\right)$ implies that the integral Hodge conjecture holds for products $C \times X$, where $C$ is a smooth curve).

Coming back to Theorem 0.9, (ii), we will even prove a stronger statement (see Theorem 1.10), namely that in the situation and with the notation above, $X$ does not satisfy property $(*)$. This in particular answers negatively the following question originally asked by Harris, de Jong and Starr, solved positively for the intersection of two quadrics [6] and for many degrees for the cubic threefold [21, [19, 28]:

Question: Let $X$ be a rationally connected threefold. Is it true that the Abel-Jacobi map on the main component of the family of rational curves of sufficiently positive class has rationally connected general fiber?

(Note that the paper [14] exhibits a totally different behaviour for rational curves on cubic fourfolds. Note also that Castravet [6] gives examples of $X$ as above, with $H_{2}(X, \mathbb{Z})=\mathbb{Z}$ 
and for which the family of free rational curves of degree $d$ is reducible for all degrees $d$, the general point of the "main component" parameterizing a very free rational curve.)

The method of the proof of Theorems 0.1 and 0.9 . (i), is by degeneration of the general quartic double solid $X_{t}$, (or the general $k$-nodal double solid $X_{t}$, ) to the Artin-Mumford nodal quartic double solid $X_{0}$. Our general result proved in Section 1 is the general specialization theorem 1.1 implying in our case that the non-existence of a decomposition of the diagonal (Chow-theoretic or cohomological) for a desingularization of $X_{0}$ implies the non-existence also for the very general smooth double solid $X_{t}$, or for the desingularization $\widetilde{X}_{t}$ of a very general double solid with $k \leq 7$ nodes. The interesting fact in this degeneration argument is the following: the desingularization of the Artin-Mumford double solid does not admit an integral cohomological decomposition of the diagonal because it has some nontrivial torsion in its integral cohomology. The desingularization of the general $k \leq 7$-nodal double solid then does not admit an integral cohomological decomposition of the diagonal, but it has no torsion anymore in its integral cohomology. The non-existence of integral cohomological decomposition of the diagonal thus implies that another property from 1 to 5 in Theorem 0.7 must be violated, and when $k=7$, the only one which can be violated is the existence of a universal codimension 2 cycle.

Thanks. I thank Jean-Louis Colliot-Thélène for sending me the very interesting paper [2, for inspiring discussions related to it and for his criticism on the exposition. I also thank the anonymous referee for his very helpful criticism and suggestions.

\section{A degeneration argument}

We prove in this section the following degeneration (or specialization) result.

Theorem 1.1. Let $\pi: \mathcal{X} \rightarrow B$ be a flat projective morphism of relative dimension $n \geq 2$, where $B$ is a smooth curve 1 . Assume that the fiber $\mathcal{X}_{t}$ is smooth for $t \neq 0$, and has at worst ordinary quadratic singularities for $t=0$. Then

(i) If for general $t \in B, \mathcal{X}_{t}$ admits a Chow theoretic decomposition of the diagonal (equivalently, $\mathrm{CH}_{0}\left(\mathcal{X}_{t}\right)$ is universally trivial), the same is true for any smooth projective model $\widetilde{\mathcal{X}}_{o}$ of $\mathcal{X}_{o}$.

(ii) If for general $t \in B, \mathcal{X}_{t}$ admits a cohomological decomposition of the diagonal, and the even degree integral homology of a smooth projective model $\widetilde{\mathcal{X}}_{o}$ of $\mathcal{X}_{o}$ is algebraic (i.e. generated over $\mathbb{Z}$ by classes of subvarieties), $\widetilde{\mathcal{X}}_{o}$ also admits a cohomological decomposition of the diagonal.

Remark 1.2. As the proof will show, the assumptions on the singularities of the central fiber can be weakened as follows: For (i), it suffices to assume that the central fiber is irreducible and admits a desingularization $\widetilde{\mathcal{X}}_{o} \rightarrow \mathcal{X}_{o}$ with smooth exceptional divisor $E$, whose connected components $E_{i}$ have universally trivial $\mathrm{CH}_{0}$ group (for example, is rational) 2 . For (ii), it would suffice to know also that with the same conditions on the desingularization, the even degree integral homology of $\widetilde{\mathcal{X}}_{o}$ is algebraic, the odd degree cohomology of $E$ is trivial and the even degree integral homology of $E$ is without torsion and generated by classes of algebraic cycles. These properties are clearly satisfied when $E$ is a disjoint union of smooth quadrics.

Remark 1.3. Theorem 1.1 will be used in applications in the following form: If the desingularization $\widetilde{\mathcal{X}}_{o}$ of the central fiber does not admit an integral Chow-theoretic or cohomological decomposition of the diagonal, the very general fiber $\mathcal{X}_{t}$ does not admit an integral Chowtheoretic or cohomological decomposition of the diagonal. This is because the set of points

\footnotetext{
${ }^{1}$ I thank J.-L. Colliot-Thélène for bringing to my attention the fact that the smoothness assumption I had originally put on $\mathcal{X}$ was in fact not used in the proof.

${ }^{2}$ A. Pirutka and J.-L. Colliot-Thélène 11 have indeed applied successfully the same method in the case of a specialization to more complicated singularities, generalizing our results to quartic threefolds, instead of quartic double solids.
} 
$t \in B_{\text {reg }}$ such that the fiber $\mathcal{X}_{t}$ is smooth and admits an integral Chow-theoretic (resp. cohomological) decomposition of the diagonal is a countable union of closed algebraic subsets of $B_{\text {reg }}$, see below.

Before giving the proof of the theorem, let us make one more remark. The proof is in two steps: The first one consists in proving that the central fiber $\mathcal{X}_{o}$ admits a decomposition of the diagonal. This follows from a specialization result due to Fulton [16] which we will reprove below for completeness. One has to be a little careful here since $\mathcal{X}_{o}$ is singular. The decomposition of the diagonal for $\mathcal{X}_{o}$ will hold in $\mathrm{CH}_{n}\left(\mathcal{X}_{o} \times \mathcal{X}_{o}\right)$. This first step does not use any assumption on the singularities of $\mathcal{X}_{o}$. The second step consists in comparing what happens for $\mathcal{X}_{o}$ and $\widetilde{\mathcal{X}}_{o}$. It is here that the assumption on the singularities is used in an essential way. Note that Theorem 1.1 is not true without assumptions on the singularities of the central fiber, as the simplest example shows: Consider a family of smooth cubic surfaces specializing to a cubic surface which is a cone over a smooth elliptic curve. Then the general fiber is rational, hence admits a Chow-theoretic decomposition of the diagonal, but the desingularization $\widetilde{\mathcal{X}}_{o}$ of the central fiber, being a $\mathbb{P}^{1}$-bundle over an elliptic curve $E$, does not admit one, even with $\mathbb{Q}$-coefficients, since $\mathrm{CH}_{0}\left(\widetilde{\mathcal{X}}_{o}\right)=\mathrm{CH}_{0}(E)_{0}$ is nontrivial.

Proof of Theorem 1.1. (i) For a general $t \in B$, there exist an effective divisor $D_{t} \subset \mathcal{X}_{t}$ and a cycle $Z_{t}$ supported on $D_{t} \times \mathcal{X}_{t}$ such that for any point $x_{t} \in \mathcal{X}_{t}$, one has

$$
\Delta_{\mathcal{X}_{t}}=Z_{t}+\mathcal{X}_{t} \times x_{t} \text { in } \mathrm{CH}^{n}\left(\mathcal{X}_{t} \times \mathcal{X}_{t}\right) .
$$

The set of data $\left(D_{t}, x_{t}, Z_{t}\right)$ above is parameterized by a countable union of algebraic varieties over $B$ whose image in $B$ contains a Zariski open set. It follows by a Baire category argument that one of these algebraic varieties dominates $B$, so that there is a smooth finite cover $B^{\prime} \rightarrow B$ and a divisor $\mathcal{D} \subset \mathcal{X}^{\prime}:=B^{\prime} \times_{B} \mathcal{X}$, which we may assume to contain no fiber of $\mathcal{X}^{\prime} \rightarrow B^{\prime}$, a section $\sigma: B^{\prime} \rightarrow \mathcal{X}^{\prime}$ and a codimension $n$ (or dimension $n+1$ ) cycle $\mathcal{Z}$ in $\mathcal{X}^{\prime} \times{ }_{B^{\prime}} \mathcal{X}^{\prime}$ supported on $\mathcal{D} \times{ }_{B^{\prime}} \mathcal{X}^{\prime}$, with the properties that for general $t \in B^{\prime}$,

$$
\Delta_{\mathcal{X}_{t}^{\prime}}=\mathcal{Z}_{t}+\mathcal{X}_{t}^{\prime} \times \sigma(t) \text { in } \mathrm{CH}_{n}\left(\mathcal{X}_{t}^{\prime} \times \mathcal{X}_{t}^{\prime}\right)
$$

where $\mathcal{Z}_{t}=\mathcal{Z}_{\mid \mathcal{X}_{t}^{\prime} \times \mathcal{X}_{t}^{\prime}} \in \mathrm{CH}_{n}\left(\mathcal{X}_{t}^{\prime} \times \mathcal{X}_{t}^{\prime}\right)$ is well-defined even if $\mathcal{X}^{\prime} \times{ }_{B^{\prime}} \mathcal{X}^{\prime}$ is singular, because $\mathcal{X}_{t}^{\prime} \times \mathcal{X}_{t}^{\prime}$ is a Cartier divisor in $\mathcal{X}^{\prime} \times_{B^{\prime}} \mathcal{X}^{\prime}$ (see [16, 2.3]). Now we use the following general fact (we include a proof for completeness):

Proposition 1.4. Let $\pi: \mathcal{Y} \rightarrow B$ be a flat morphism of algebraic varieties, where $B$ is smooth of dimension $r$, and let $\mathcal{Z} \in \mathrm{CH}_{N}(\mathcal{Y})$ be a cycle. Then the set $B_{Z}$ of points $t \in B$ such that $\mathcal{Z}_{t}:=\mathcal{Z}_{\mid \mathcal{Y}_{t}}$ vanishes in $\mathrm{CH}_{N-r}\left(\mathcal{Y}_{t}\right)$ is a countable union of proper closed algebraic subsets of $B$.

Proof. For any $t \in B$ such that $\mathcal{Z}$ and $\mathcal{Y}_{t}$ intersect properly and $\mathcal{Z}_{\mid \mathcal{Y}_{t}}=0$ in $\mathrm{CH}_{N-r}\left(\mathcal{Y}_{t}\right)$, there exist subvarieties $W_{i, t} \subset \mathcal{Y}_{t}$ of dimension $N-r+1$ and nonzero rational functions $\phi_{i, t}$ on $\widetilde{W_{i, t}} \stackrel{j_{i, t}}{\rightarrow} \mathcal{Y}_{t}$ such that $\sum_{i} j_{i, t_{*}} \operatorname{div} \phi_{i, t}=\mathcal{Z}_{\mid \mathcal{Y}_{t}}$. The data $\left(W_{i, t}, \phi_{i, t}\right)$ are parameterized by a countable union of quasi-projective irreducible varieties $M_{l} \stackrel{\alpha_{l}}{\rightarrow} B$, whose image in $B$ is exactly the set $B_{Z}$. We may assume that the $M_{l}$ 's are smooth. For each of these varieties $M_{l}$, there exists a closed algebraic subvariety $B_{l} \subset B$, such that $\operatorname{Im} \alpha_{l}$ contains a Zariski open set $B_{l}^{0}$ of $B_{l}$. Let $B_{l}^{0}=\alpha_{l}\left(M_{l}^{0}\right)$. For any point $t$ of $B_{l}^{0}$, we have $\mathcal{Z}_{\mid \mathcal{Y}_{t}}=0$ in $\mathrm{CH}_{N-r}\left(\mathcal{Y}_{t}\right)$ and it only remains to show that this remains true for any point of $B_{l}$, since we then have $B_{Z}=\cup_{l} B_{l}$. We observe now that we can assume that the $M_{l}$ 's carry universal objects, and thus that the cycle $\mathcal{Z}_{l}:=\alpha_{l}^{\prime *} \mathcal{Z}$ is rationally equivalent to 0 on $\mathcal{Y}_{M_{l}}^{\prime}$, where

$$
\mathcal{Y}_{M_{l}}^{\prime}:=M_{l} \times_{B} \mathcal{Y} \stackrel{\pi^{\prime}}{\rightarrow} M_{l},
$$

and $\alpha_{l}^{\prime}: \mathcal{Y}_{M_{l}}^{\prime} \rightarrow \mathcal{Y}$ is the natural map. Here we use the fact, which will be used again below, that the morphism

$$
\alpha_{l}^{\prime *}: \mathrm{CH}_{N}(\mathcal{Y}) \rightarrow \mathrm{CH}_{N^{\prime}}\left(\mathcal{Y}_{M_{l}}^{\prime}\right), N^{\prime}=N-r+\operatorname{dim} M_{l}
$$


is well-defined, because $B$ is smooth and $\mathcal{Y} \rightarrow B$ is flat. Let now $\overline{M_{l}}$ be a smooth partial completion of $M_{l}$ on which the morphism $\alpha_{l}$ extends to a projective morphism $\bar{\alpha}_{l}: \overline{M_{l}} \rightarrow B$ whose image is equal to $B_{l}$ by properness. Denote by $\overline{\mathcal{Y}_{M_{l}}^{\prime}}$ the fibered product $\overline{M_{l}} \times{ }_{B} \mathcal{Y}$, with natural morphism $\pi^{\prime \prime}: \overline{\mathcal{Y}_{M_{l}}^{\prime}} \rightarrow \overline{M_{l}}$ extending $\pi^{\prime}$, and by $\overline{\alpha_{l}^{\prime}}: \overline{\mathcal{Y}_{M_{l}}^{\prime}} \rightarrow \mathcal{Y}$ the natural map.

Then $\operatorname{Im} \bar{\alpha}_{l}=B_{l}$, and for any $s \in \overline{M_{l}}$ with image $t \in B_{l}$, we have

$$
\bar{\alpha}_{l}^{\prime *}(\mathcal{Z}){\overline{\mathcal{Y}_{M_{l} s}^{\prime}}}^{\prime}=\mathcal{Z}_{\mid \mathcal{Y}_{t}} \text { in } \mathrm{CH}_{N-r}\left(\mathcal{Y}_{t}\right)
$$

As the cycle $\bar{\alpha}_{l}^{\prime *}(\mathcal{Z})$ vanishes on the Zariski open set $\pi^{\prime \prime-1}\left(M_{l}^{0}\right)=\pi^{\prime-1}\left(M_{l}^{0}\right)$, we thus conclude applying to $M=\bar{M}_{l}, W=\overline{\mathcal{Y}_{M_{l}}^{\prime}}, f=\pi^{\prime \prime}, \Gamma=\bar{\alpha}_{l}^{\prime *}(\mathcal{Z})$ the following lemma:

Lemma 1.5. Let $M$ be smooth of dimension $m$ and let $f: W \rightarrow M$ be a flat morphism. Let $\Gamma$ be a $N$-cycle on $W$. Assume there is a Zariski dense open set $M^{0}$ of $M$ such that $\Gamma_{\mid W^{0}}=0$ in $\mathrm{CH}\left(W^{0}\right)$, where $W^{0}:=f^{-1}\left(M^{0}\right)$. Then for any $t \in M, \Gamma_{\mid W_{t}}=0$ in $\mathrm{CH}_{N-m}\left(W_{t}\right)$.

Proof. We may assume that $M$ is a smooth curve. Let $D$ be the divisor $M \backslash M^{0} \subset M$. By the localization exact sequence, there is a $N$-cycle $z$ supported on $f^{-1}(D)$ such that

$$
\Gamma=i_{*}(z) \text { in } \mathrm{CH}_{N}(W),
$$

where $i$ is the inclusion of the Cartier divisor $f^{-1}(D)$ in $W$. The fact that $\Gamma_{\mid W_{t}}=0$ in $\mathrm{CH}_{N-m}\left(W_{t}\right)$ for any $t \in D$ then follows from the fact that $f^{-1}(D)_{\mid W_{t}}$ is the trivial Cartier divisor on $W_{t}$, and from Fulton's definition of the intersection with a Cartier divisor, which says that for any point of $t \in D, i_{*}(z)_{\mid W_{t}}=f^{-1}(D)_{\mid W_{t}} \cdot z_{t}$, where $z_{t}$ is the part of $z$ lying in the component $W_{t}$ of $f^{-1}(D)$.

It follows that for any $t \in \overline{M_{l}}$, the restriction of the cycle ${\bar{\alpha}_{l}^{\prime}}^{*}(\mathcal{Z})$ to the fiber $\pi^{\prime-1}(t) \subset$ $\overline{\mathcal{Y}_{M_{l}}^{\prime}}$ is trivial, and this implies that for any $t \in B_{l}$, the restriction of the cycle $\mathcal{Z}$ to the fiber $\pi^{-1}(t) \subset \mathcal{Y}_{t}$ is trivial.

By Proposition 1.4, the locus of points $t \in B^{\prime}$ such that (6) holds is a countable union of closed algebraic subsets of $B^{\prime}$. As it contains by assumption a Zariski open set, we conclude that (6) holds for any $t \in B^{\prime}$. Choose for $t$ any point $o^{\prime}$ over $o \in B$. Then identifying $\mathcal{X}_{o^{\prime}}^{\prime}$ with $\mathcal{X}_{o}$ we conclude that there exist a divisor $D_{o} \subset \mathcal{X}_{o}$, a point $x_{o} \in \mathcal{X}_{o}$ and a cycle $Z_{o}$ supported on $D_{o} \times \mathcal{X}_{o}$, such that

$$
\Delta_{\mathcal{X}_{o}}=Z_{o}+\mathcal{X}_{o} \times x_{o} \text { in } \mathrm{CH}_{n}\left(\mathcal{X}_{o} \times \mathcal{X}_{o}\right) .
$$

Let $\tau: \widetilde{\mathcal{X}}_{o} \rightarrow \mathcal{X}_{o}$ be the desingularization obtained by blowing-up the singular points. It remains to deduce from (7) that $\widetilde{\mathcal{X}}_{o}$ satisfies the same property. Let $x_{i}, i=1, \ldots, N$ be the singular points of $\mathcal{X}_{o}$, and $E_{i}:=\tau^{-1}\left(x_{i}\right)$. By assumption, $E_{i}$ is a smooth quadric of dimension $\geq 1$, and in particular $E_{i}$ is rational and has universally trivial $\mathrm{CH}_{0}$ group. Let $E:=\cup_{i} E_{i}$. Then (7) restricted to

$$
\left(\mathcal{X}_{o} \backslash\left\{x_{1}, \ldots, x_{N}\right\}\right) \times\left(\mathcal{X}_{o} \backslash\left\{x_{1}, \ldots, x_{N}\right\}\right) \cong\left(\widetilde{\mathcal{X}}_{o} \backslash E\right) \times\left(\widetilde{\mathcal{X}}_{o} \backslash E\right)
$$

provides:

$$
\Delta_{\tilde{\mathcal{X}}_{o} \backslash E}=Z_{o}+\left(\widetilde{\mathcal{X}}_{o} \backslash E\right) \times x_{o} \text { in } \mathrm{CH}_{n}\left(\left(\widetilde{\mathcal{X}}_{o} \backslash E\right) \times\left(\widetilde{\mathcal{X}}_{o} \backslash E\right)\right) .
$$

where $Z_{o}$ is supported on $D_{o} \times\left(\widetilde{\mathcal{X}}_{o} \backslash E\right)$ for a proper closed algebraic subset $D_{o}$ of $\widetilde{\mathcal{X}}_{o} \backslash E$. The localization exact sequence allows to rewrite (8) as

$$
\Delta_{\widetilde{\mathcal{X}}_{o}}=Z_{o}+\widetilde{\mathcal{X}}_{o} \times x_{o}+Z \text { in } \mathrm{CH}_{n}\left(\tilde{\mathcal{X}}_{o} \times \widetilde{\mathcal{X}}_{o}\right) .
$$


where $Z_{o}$ is supported on $D_{o}^{\prime} \times \widetilde{\mathcal{X}}_{o}$ for a proper closed algebraic subset $D_{o}^{\prime} \varsubsetneqq \widetilde{\mathcal{X}}_{o}$ and $Z$ is supported on $\widetilde{\mathcal{X}}_{o} \times E \cup E \times \widetilde{\mathcal{X}}_{o}$. Writing $Z$ as $Z_{1}+Z_{2}$, where $Z_{1}$ is supported on $E \times \widetilde{\mathcal{X}}_{o}$, and $Z_{2}$ is supported on $\widetilde{\mathcal{X}}_{o} \times E$, it is clear that up to replacing in (9) $Z_{o}$ by $Z_{o}+Z_{1}$ and $D_{o}^{\prime}$ by $D_{o}^{\prime} \cup E$, we may assume that $Z=Z_{2}$ is supported on

$$
\widetilde{\mathcal{X}}_{o} \times E=\bigsqcup_{i} \widetilde{\mathcal{X}}_{o} \times E_{i}
$$

Let $Z_{2, i}$ be the restriction of $Z_{2}$ to the connected component $\widetilde{\mathcal{X}}_{o} \times E_{i}$. As $\operatorname{dim} Z_{2}=n=$ $\operatorname{dim} \widetilde{\mathcal{X}}_{o}$ and $E_{i}$ has universally trivial $\mathrm{CH}_{0}$ group, one can write for each $i$

$$
Z_{2, i}=Z_{2, i}^{\prime}+\mu_{i} \widetilde{\mathcal{X}}_{o} \times x_{i}
$$

in $\mathrm{CH}_{n}\left(\widetilde{\mathcal{X}}_{o} \times E_{i}\right)$, where $x_{i}$ is a point of $E_{i}, \mu_{i} \in \mathbb{Z}$ and $Z_{2, i}^{\prime}$ is supported on $D_{i} \times E_{i}$ for some proper closed algebraic subset $D_{i}$ of $\widetilde{\mathcal{X}}_{o}$.

Combining (9) and (10), we finally conclude that

$$
\Delta_{\widetilde{\mathcal{X}}_{o}}=Z_{o}^{\prime}+\widetilde{\mathcal{X}}_{o} \times x_{o}+\sum_{i} \mu_{i} \widetilde{\mathcal{X}}_{o} \times x_{i} \text { in } \mathrm{CH}^{n}\left(\widetilde{\mathcal{X}}_{o} \times \widetilde{\mathcal{X}}_{o}\right),
$$

where $Z_{o}^{\prime}=Z_{o}+Z_{1}+\sum_{i} Z_{2, i}^{\prime}$ is supported on $D_{o}^{\prime \prime} \times \widetilde{\mathcal{X}}_{o}$ for a proper closed algebraic subset $D_{o}^{\prime \prime}=D_{o}^{\prime} \cup E \cup\left(\cup_{i} D_{i}\right)$ of $\widetilde{\mathcal{X}}_{o}$. Formula (11) implies that $\sum_{i} \mu_{i}=0$ and that $\mathrm{CH}_{0}\left(\widetilde{\mathcal{X}}_{o}\right)$ is generated by the $x_{i}, i=o, 1, \ldots, N$, which easily implies that $\mathrm{CH}_{0}\left(\widetilde{\mathcal{X}}_{o}\right)=\mathbb{Z}$ since the central fiber is irreducible under our assumptions (this is why we impose the condition that the fiber dimension is $\geq 2$; in fact, Theorem 1.1 is wrong if the fiber dimension is 1 , because the disjoint union of two $\mathbb{P}^{1}$ does not admit a Chow-theoretic decomposition of the diagonal). Hence (11) gives

$$
\Delta_{\widetilde{\mathcal{X}}_{o}}=Z_{o}^{\prime}+\widetilde{\mathcal{X}}_{o} \times x_{o} \text { in } \mathrm{CH}^{n}\left(\widetilde{\mathcal{X}}_{o} \times \widetilde{\mathcal{X}}_{o}\right),
$$

which concludes the proof of (i).

(ii) The proof of (ii) works very similarly. We first construct as before a smooth finite cover $B^{\prime} \rightarrow B$, a divisor $\mathcal{D} \subset \mathcal{X}^{\prime}:=B^{\prime} \times_{B} \mathcal{X}$, which we may assume to contain no fiber of $\mathcal{X}^{\prime} \rightarrow B^{\prime}$, a section $\sigma: B^{\prime} \rightarrow \mathcal{X}^{\prime}$ and a codimension $n$ cycle $\mathcal{Z}$ in $\mathcal{X}^{\prime} \times{ }_{B^{\prime}} \mathcal{X}^{\prime}$ supported on $\mathcal{D} \times{ }_{B^{\prime}} \mathcal{X}^{\prime}$, with the properties that for general $t \in B^{\prime}$ (so in particular $\mathcal{X}_{t}^{\prime}$ is smooth), we have the equality of cycle classes

$$
\left[\Delta_{\mathcal{X}_{t}^{\prime}}\right]=\left[\mathcal{Z}_{t}\right]+\left[\mathcal{X}_{t}^{\prime} \times \sigma(t)\right] \text { in } H^{2 n}\left(\mathcal{X}_{t}^{\prime} \times \mathcal{X}_{t}^{\prime}, \mathbb{Z}\right)=H_{2 n}\left(\mathcal{X}_{t}^{\prime} \times \mathcal{X}_{t}^{\prime}, \mathbb{Z}\right)
$$

where $\mathcal{Z}_{t}=\mathcal{Z}_{\mid \mathcal{X}_{t}^{\prime} \times \mathcal{X}_{t}^{\prime}}$. We now work in the analytic setting and restrict to $\mathcal{X}_{\Delta}^{\prime}$, where $\Delta$ is a small disc in $B^{\prime}$ centered at a point $o^{\prime}$ of $B^{\prime}$ over $o \in B$, such that $\mathcal{X}_{\Delta}^{\prime}$ retracts continuously for the usual topology on the central fiber $\mathcal{X}_{o^{\prime}}^{\prime}$, (the retraction map being homotopic over $\Delta$ to the identity,) so that $\mathcal{X}_{\Delta}^{\prime} \times_{\Delta} \mathcal{X}_{\Delta}^{\prime}$ retracts similarly on $\mathcal{X}_{o^{\prime}}^{\prime} \times \mathcal{X}_{o^{\prime}}^{\prime} \cong \mathcal{X}_{o} \times \mathcal{X}_{o}$. Then we conclude that (12) implies

$$
\left[\Delta_{\mathcal{X}_{o}}\right]=\left[\mathcal{Z}_{o}\right]+\left[\mathcal{X}_{o} \times x_{o}\right] \text { in } H_{2 n}\left(\mathcal{X}_{o} \times \mathcal{X}_{o}, \mathbb{Z}\right),
$$

where the cycle classes are from now on taken in homology. This is because the topological retraction from $\mathcal{X}_{\Delta}^{\prime} \times_{\Delta} \mathcal{X}_{\Delta}^{\prime}$ to $\mathcal{X}_{o^{\prime}}^{\prime} \times \mathcal{X}_{o^{\prime}}^{\prime}$ induces an isomorphism

$$
H_{*}\left(\mathcal{X}_{o^{\prime}}^{\prime} \times \mathcal{X}_{o^{\prime}}^{\prime}, \mathbb{Z}\right) \cong H_{*}\left(\mathcal{X}_{\Delta}^{\prime} \times_{\Delta} \mathcal{X}_{\Delta}^{\prime}, \mathbb{Z}\right)
$$

and by flatness, the classes

$$
\left[\Delta_{\mathcal{X}_{t}^{\prime}}\right],\left[\mathcal{Z}_{t}\right] \in H_{2 n}\left(\mathcal{X}_{\Delta}^{\prime} \times_{\Delta} \mathcal{X}_{\Delta}^{\prime}, \mathbb{Z}\right)
$$


are constant (that is, independent of $t$ ) in

$$
H_{2 n}\left(\mathcal{X}_{\Delta}^{\prime} \times_{\Delta} \mathcal{X}_{\Delta}^{\prime}, \mathbb{Z}\right)=H_{2 n}\left(\mathcal{X}_{o^{\prime}}^{\prime} \times \mathcal{X}_{o^{\prime}}^{\prime}, \mathbb{Z}\right) .
$$

With the same notation $\widetilde{\mathcal{X}}_{o}, x_{i}, E_{i}, E$ as in the proof of (i), we deduce from (13) by taking the image in the relative homology of the pair $\left(\mathcal{X}_{o} \times \mathcal{X}_{o},\left(\mathcal{X}_{o} \times\left\{x_{1}, \ldots x_{N}\right\} \cup\left\{x_{1}, \ldots x_{N}\right\} \times \mathcal{X}_{o}\right)\right)$ the following equality in the relative homology group

$$
\begin{gathered}
H_{2 n}\left(\mathcal{X}_{o} \times \mathcal{X}_{o},\left(\mathcal{X}_{o} \times\left\{x_{1}, \ldots x_{N}\right\} \cup\left\{x_{1}, \ldots x_{N}\right\} \times \mathcal{X}_{o}\right), \mathbb{Z}\right)=H_{2 n}\left(\widetilde{\mathcal{X}}_{o} \times \widetilde{\mathcal{X}}_{o},\left(\widetilde{\mathcal{X}}_{o} \times E \cup E \times \widetilde{\mathcal{X}}_{o}\right), \mathbb{Z}\right) \\
{\left[\Delta_{\tilde{\mathcal{X}}_{o}}\right]_{r e l}=\left[\mathcal{Z}_{o}\right]_{r e l}+\left[\mathcal{X}_{o} \times x_{o}\right]_{r e l} \text { in } H_{2 n}\left(\widetilde{\mathcal{X}}_{o} \times \widetilde{\mathcal{X}}_{o},\left(\widetilde{\mathcal{X}}_{o} \times E \cup E \times \widetilde{\mathcal{X}}_{o}\right), \mathbb{Z}\right),}
\end{gathered}
$$

where the subscript "rel" indicates that we consider the homology class in the relative homology group $H_{2 n}\left(\widetilde{\mathcal{X}}_{o} \times \widetilde{\mathcal{X}}_{o},\left(\widetilde{\mathcal{X}}_{o} \times E \cup E \times \widetilde{\mathcal{X}}_{o}\right), \mathbb{Z}\right)$. Formula (14) and the long exact sequence of relative homology imply that the homology class

$$
\left[\Delta_{\tilde{\mathcal{X}}_{o}}\right]-\left[\mathcal{Z}_{o}\right]-\left[\widetilde{\mathcal{X}}_{o} \times x_{o}\right] \in H_{2 n}\left(\tilde{\mathcal{X}}_{o} \times \widetilde{\mathcal{X}}_{o}, \mathbb{Z}\right)
$$

comes from a homology class

$$
\beta \in H_{2 n}\left(\widetilde{\mathcal{X}}_{o} \times E \cup E \times \widetilde{\mathcal{X}}_{o}, \mathbb{Z}\right) .
$$

Note now that the closed subset

$$
\widetilde{\mathcal{X}}_{o} \times E \cup E \times \widetilde{\mathcal{X}}_{o} \subset \widetilde{\mathcal{X}}_{o} \times \widetilde{\mathcal{X}}_{o}
$$

is the union of $\widetilde{\mathcal{X}}_{o} \times E$ and $E \times \widetilde{\mathcal{X}}_{o}$ glued along $E \times E$. We thus have a Mayer-Vietoris exact sequence

$$
\ldots H_{2 n}\left(\widetilde{\mathcal{X}}_{o} \times E, \mathbb{Z}\right) \oplus H_{2 n}\left(E \times \widetilde{\mathcal{X}}_{o}, \mathbb{Z}\right) \rightarrow H_{2 n}\left(\widetilde{\mathcal{X}}_{o} \times E \cup E \times \widetilde{\mathcal{X}}_{o}, \mathbb{Z}\right) \rightarrow H_{2 n-1}(E \times E, \mathbb{Z}) \rightarrow \ldots
$$

As $E \times E=\bigsqcup_{i, j} E_{i} \times E_{j}$ and $E_{i} \times E_{j}$ has trivial homology in odd degree, we conclude that $H_{2 n-1}(E \times E, \mathbb{Z})=0$, so that $\beta$ comes from a homology class

$$
\gamma=\left(\gamma_{1}, \gamma_{2}\right) \in H_{2 n}\left(\widetilde{\mathcal{X}}_{o} \times E, \mathbb{Z}\right) \oplus H_{2 n}\left(E \times \widetilde{\mathcal{X}}_{o}, \mathbb{Z}\right)=H^{2 n-2}\left(\widetilde{\mathcal{X}}_{o} \times E, \mathbb{Z}\right) \oplus H^{2 n-2}\left(E \times \widetilde{\mathcal{X}}_{o}, \mathbb{Z}\right)
$$

We now use the assumption made on $\widetilde{\mathcal{X}}_{o}$, namely that its even degree cohomology is algebraic. As the cohomology of $E$ has no torsion and is algebraic, we get by the Künneth decomposition that

$$
H^{2 n-2}\left(E \times \widetilde{\mathcal{X}}_{o}, \mathbb{Z}\right)=\oplus_{0 \leq 2 i \leq 2 n-2} H^{2 i}(E, \mathbb{Z}) \otimes H^{2 n-2-2 i}\left(\widetilde{\mathcal{X}}_{o}, \mathbb{Z}\right)
$$

is generated by classes of algebraic cycles $z_{j} \times z_{j}^{\prime} \subset E \times \tilde{\mathcal{X}}_{o}$ and similarly for $\tilde{\mathcal{X}}_{o} \times E$.

Putting everything together, we get an equality

$$
\left[\Delta_{\tilde{\mathcal{X}}_{o}}\right]-\left[\mathcal{Z}_{o}\right]-\left[\mathcal{X}_{o} \times x_{o}\right]=\sum_{j} n_{j}\left[z_{j} \times z_{j}^{\prime}\right]+\sum_{k} n_{k}^{\prime}\left[z_{k}^{\prime} \times z_{k}\right] \text { in } H_{2 n}\left(\widetilde{\mathcal{X}}_{o} \times \widetilde{\mathcal{X}}_{o}, \mathbb{Z}\right)
$$

This provides us with an integral cohomological decomposition of the diagonal for $\widetilde{\mathcal{X}}_{o}$ since in the term on the right, all the cycle classes of the form $\left[\widetilde{\mathcal{X}}_{o} \times\right.$ point $]$ are cohomologous and they have to sum-up to zero, while all the other terms $\left[z_{k}^{\prime} \times z_{k}\right]$ with $\operatorname{dim} z_{k}^{\prime}<n$ are supported on $D \times \widetilde{\mathcal{X}}_{o}$ for some proper closed algebraic subset $D$ of $\widetilde{\mathcal{X}}_{o}$.

Let us now deduce from Theorem 1.1 the following result: 
Theorem 1.6. (cf. Theorem 0.1 and Theorem [0.9, (i)) Let $\widetilde{X}$ be the natural desingularization of a very general quartic double solid $X$ with $k \leq 7$ nodes. Then the integral cohomology of $\widetilde{X}$ has no torsion, but $\widetilde{X}$ does not admit an integral cohomological decomposition of the diagonal. A fortiori, $\widetilde{X}$ does not admit a Chow-theoretic decomposition of the diagonal, that is, equivalently, the group $\mathrm{CH}_{0}(\widetilde{X})$ is not universally trivial.

Proof. The first statement is proved in [15, if we observe in the nodal case that $\widetilde{X}$ admits a unirational parametrization of degree 2 (as all nodal quartic double solids do, see paragraph 0.1). This indeed implies that the torsion in $H^{*}(\widetilde{X}, \mathbb{Z})$ is of order 2, while Endrass $[15$, proves that there is no 2-torsion in $H^{*}(\widetilde{X}, \mathbb{Z})$ if $\widetilde{X}$ has less than 10 nodes.

We next claim the following:

Lemma 1.7. The general quartic double solid $X$ with $k \leq 7$ nodes can be specialized to the Artin-Mumford double solid $X_{o}$ constructed in [1]. In particular, its desingularization obtained by blowing-up its $k$ nodes can be specialized onto the partial desingularization of $X_{o}$ obtained by blowing-up the corresponding $k$ nodes.

Postponing the proof of the lemma, we now conclude as follows: First of all, as $\widetilde{X}_{o}$ has by Artin-Mumford [1] some nontrivial 2-torsion in its integral cohomology, it does not admit an integral cohomological decomposition of the diagonal (see [28] or Theorem 0.7). We use now 27] which guarantees that the even degree integral cohomology of $\widetilde{X}_{o}$ is algebraic, because $\widetilde{X}_{o}$ is uniruled of dimension 3. It then follows from Lemma 1.7 and Theorem 1.1 that the very general $\widetilde{X}$ as in Corollary 1.6 does not admit an integral cohomological decomposition of the diagonal.

Proof of Lemma 1.7. The data of a $k$-nodal quartic double solid is equivalent to the data of the corresponding quartic ramification surface which is also $k$-nodal. Let us consider a general Artin-Mumford quartic surface $S$. It has 10 nodes $P_{0}, \ldots, P_{9}$, where $P_{0}$ is the point defined in coordinates $X_{0}, \ldots, X_{3}$ by $X_{0}=X_{1}=X_{2}=0$ and the $P_{i}, i \geq 1$ are above 9 points $O_{i} \in \mathbb{P}^{2}$ via the linear projection $\mathbb{P}^{3} \rightarrow \mathbb{P}^{2}$ from $P_{0}$. The 9 points $O_{i}, i \geq 1$ form the reduced intersection of two plane cubics. The general deformation theory of $K 3$ surfaces (see [33]) tells us that the Artin-Mumford surface with 10 nodes $P_{0}, \ldots, P_{9}$ can have its nodes smoothed independently, keeping the other nodes. Let us prove this statement in a more algebraic and elementary way.

Sublemma 1.8. For any $k \leq 10$, there exist a smooth quasiprojective variety $B$, and a family of quartic surfaces $\pi: \mathcal{S} \rightarrow B, \mathcal{S} \subset B \times \mathbb{P}^{3}$ with the following property: The general fiber of $\pi$ is $k$-nodal and there exists a non-empty proper closed algebraic subset $B^{\prime} \subset B$ of codimension $10-k$ parameterizing 10-nodal Artin-Mumford surface 3 .

Proof. First of all, we claim that if $S \subset \mathbb{P}^{3}$ is a $s+1$-nodal quartic surface defined by a quartic polynomial $f$, then the set $Z$ of nodes $P_{i}, i=0, \ldots, s$ of $S$ imposes $s+1$ independent conditions to quartic polynomials. Indeed, one easily check that there exists an irreducible nodal curve in the linear system $\left|\mathcal{I}_{Z}(4)\right|$ (since $\left|\mathcal{I}_{Z}(3)\right|$ contains the partial derivatives of $f$, this linear system has no base point on $S \backslash Z$ and cuts $Z$ schematically, hence it is nef on the blow-up $\widetilde{S}$ of $S$ along $Z$; thus $\left|\mathcal{I}_{Z}(4)\right|$ is nef and big on $\left.\widetilde{S}\right)$. Then the normalized curve $n: \widetilde{C} \rightarrow C$ contains the set $\widetilde{Z}=n^{-1}(Z)$, and we have $K_{\widetilde{C}}=n^{*}\left(\mathcal{O}_{C}(4)\right)(-\widetilde{Z})$; thus, as $\widetilde{C}$ is irreducible, we have $g(\widetilde{C})=g(C)-|Z|$, which provides

$$
h^{0}\left(\widetilde{C}, n^{*}\left(\mathcal{O}_{C}(4)\right)(-\widetilde{Z})\right)=g(\widetilde{C})=g(C)-|Z|=h^{0}\left(\mathcal{O}_{C}(4)\right)-|Z| .
$$

As $H^{0}\left(\widetilde{C}, n^{*}\left(\mathcal{O}_{C}(4)\right)(-\widetilde{Z})\right)$ contains $H^{0}\left(C, \mathcal{O}_{C}(4) \otimes \mathcal{I}_{Z}\right)$, one concludes that

$$
h^{0}\left(C, \mathcal{O}_{C}(4) \otimes \mathcal{I}_{Z}\right) \leq h^{0}\left(\mathcal{O}_{C}(4)\right)-|Z|
$$

\footnotetext{
${ }^{3}$ One can even show that the total space $\mathcal{S}$ is smooth, but this is not useful here.
} 
which proves the claim.

This implies classically that in the projective space $\mathbb{P}^{N}$ of all quartic homogeneous polynomials on $\mathbb{P}^{3}$, the hypersurface $\mathcal{D}$ consisting of quartic polynomials with one node has $s+1$ (in our case, 10) analytic smooth branches intersecting transversally at $f$. Concretely, the normalization $\widetilde{\mathcal{D}}$ of $\mathcal{D}$ is defined as the subvariety of $\mathbb{P}^{3} \times \mathbb{P}^{N}$ defined by

$$
\widetilde{\mathcal{D}}=\left\{(x, g) \in \mathbb{P}^{3} \times \mathbb{P}^{N}, g \text { is singular at } x\right\},
$$

and the branches $\mathcal{D}_{i}$ of $\mathcal{D}$ passing through $f$ are in one to one correspondence with the preimages of $\widetilde{\mathcal{D}} \rightarrow \mathcal{D}$ over $f$, that is the nodes $P_{i}$ of $S$.

Coming back to our situation, the nodes $P_{1}, \ldots, P_{k}$ being fixed, the intersection of the corresponding analytic branches $\mathcal{D}_{i}, i=1, \ldots, k$ is smooth and we can construct a smooth algebraic variety $B$ containing it as an analytic open set as follows: $B$ will be an adequate Zariski open neighborhood of $\left(P_{1}, \ldots, P_{k}, f\right)$ in the set

$$
\left\{\left(x_{1}, \ldots, x_{k}, g\right) \in \mathbb{P}^{3} \times \mathbb{P}^{N}, g \text { is singular at } x_{i}, \text { for } i=1, \ldots, k\right\} .
$$

This variety $B$ maps naturally by the second projection to $\mathbb{P}^{N}$, and the pull-back to $B$ of the universal family $\mathcal{S}_{\text {univ }} \subset \mathbb{P}^{N} \times \mathbb{P}^{3}$ provides us with a family

$$
\mathcal{S} \rightarrow B, \mathcal{S} \subset \mathbb{P}^{3} \times B
$$

of quartic $K 3$ surfaces, such that the general fiber $\mathcal{S}_{b}$ has $k$ nodes $P_{1, b}, \ldots, P_{k, b}$, while the fibers $\mathcal{S}_{b_{0}}$ for $b_{0} \in B^{\prime} \varsubsetneqq B$ are Artin-Mumford quartics with 10 nodes, $k$ of them being the specialization of $P_{1, b}, \ldots, P_{k, b}$. Here, with the notation just introduced, the image of $B^{\prime}$ in $\mathbb{P}^{N}$ is the Zariski closure of the intersection $\cap_{i=0}^{9} \mathcal{D}_{i}$ of all branches. The argument above shows that the family is furthermore complete, that is, has $34-k$ parameters.

Recall now from Remark 0.2 that there is an unique irreducible component $M$ of the space $M^{\prime}$ of nodal quartic surfaces in $\mathbb{P}^{3}$ with $k \leq 7$ nodes, dominating $\left(\mathbb{P}^{3}\right)^{(k)}$ by the map $\phi: M \rightarrow\left(\mathbb{P}^{3}\right)^{(k)}$ which to a $k$-nodal quartic associates its set of nodes. Denote by $\psi: B \rightarrow M^{\prime}$ the classifying map, where $B$ is as in Sublemma 1.8 In order to prove that $\psi(B)$ is Zariski open in $M$, which is the content of the lemma, it suffices to show that the map $\phi \circ \psi: B \rightarrow\left(\mathbb{P}^{3}\right)^{(k)}$ is dominating. Let us do it for $k=7$, the other cases being similar and easier. We have the following:

Sublemma 1.9. Let $S_{t}$ be a small general deformation of $S$ with 7 nodes $P_{1, t}, \ldots, P_{7, t}$. Then the set of quartic surfaces which are singular at all the points $P_{i, t}$ is of dimension 6.

Proof. It suffices to prove the statement when $S_{t}$ is very general. First of all we claim that the Galois group of the cover $\Sigma \rightarrow M$ parameterizing the nine singular points $O_{i}$ of the surface $S_{m}, m \in M$, where $M$ is the parameter space for Artin-Mumford quartic surfaces, acts on the set of 9 points $\left\{O_{1}, \ldots, O_{9}\right\}$ as the full symmetric group. This fact can be proved by applying Harris' principle in [18. Namely, one just has to prove the following points:

1) The Galois group of the function field of $M$ acts bitransitively on the set $\left\{O_{1}, \ldots, O_{9}\right\}$. Equivalently, the variety $\Sigma \times_{M} \Sigma \backslash \Delta_{\Sigma}$ is irreducible.

2) The image contains transpositions, which appear as the local monodromy of the cover $\Sigma \rightarrow M$ at a point of simple ramification.

The variety $M$ parameterizes the triples $\left(E_{1}, E_{2}, C\right)$, where $E_{1}$ and $E_{2}$ are plane cubics, and $C$ is a conic everywhere tangent to $E_{1}$ and $E_{2}$ (see [1]). The variety $\Sigma$ parameterizes the quadruples $\left(O, E_{1}, E_{2}, C\right)$ where $\left(E_{1}, E_{2}, C\right) \in M$ and $O$ is one of the intersection points of $E_{1}$ and $E_{2}$. Let us fix $C$ and the degree 3 divisors $D_{1}$ such that $2 D_{1}=E_{1} \cap C, D_{2}$ such that $2 D_{2}=E_{2} \cap C$ of $C$. Then one gets a subvariety $M_{C, D_{1}, D_{2}} \subset M$ and its inverse image $\Sigma_{C, D_{1}, D_{2}} \subset \Sigma$. It clearly suffices to proves 1) and 2) for the general cover $\Sigma_{C, D_{1}, D_{2}} \rightarrow$ $M_{C, D_{1}, D_{2}}$. For the point 1), we project $\Sigma_{C, D_{1}, D_{2}} \times_{M_{C, D_{1}, D_{2}}} \Sigma_{C, D_{1}, D_{2}} \backslash \Sigma_{C, D_{1}, D_{2}}$ to $\mathbb{P}^{2} \times \mathbb{P}^{2}$ by the map $p$ which to $\left(\left(O, E_{1}, E_{2}, C\right),\left(O^{\prime}, E_{1}, E_{2}, C\right)\right)$ associates $\left(O, O^{\prime}\right)$. Observe now that $M_{C, D_{1}, D_{2}}$ is a Zariski open set in $\mathbb{A}^{3} \times \mathbb{A}^{3}$, the general element being of the form 
$\left(e_{1}+x c, e_{2}+y c\right)$ where $c$ is the equation of $C, e_{1}$ is given so that the restriction of $e_{1}$ to $C$ has divisor $2 D_{1}, e_{2}$ is given so that the restriction of $e_{2}$ to $C$ has divisor $2 D_{2}$, and $x, y$ are two arbitrary homogeneous polynomials of degree 1 on $\mathbb{P}^{2}$. The fiber of $p$ over a general couple of points $\left(O, O^{\prime}\right)$ then consists of the set of equations $\left(e_{1}+x c, e_{2}+y c\right)$ such that $e_{1}+x c$ and $e_{2}+y c$ vanish on $O$ and $O^{\prime}$. This gives a system of four affine equations which has maximal rank except if $O$ or $O^{\prime}$ belongs to $C$. But of course, the last situation does not occur generically on $\Sigma_{C, D_{1}, D_{2}}$, hence we conclude that for a dense Zariski open set $\Sigma_{C, D_{1}, D_{2}}^{0}$ of $\Sigma_{C, D_{1}, D_{2}}, \Sigma_{C, D_{1}, D_{2}}^{0} \times_{M} \Sigma_{C, D_{1}, D_{2}}^{0} \backslash \Delta_{\Sigma_{C, D_{1}, D_{2}}^{0}}$ is irreducible.

For the point 2), as the equations above clearly show that $\Sigma_{C, D_{1}, D_{2}}$ is smooth at a point $(O, x, y)$ where $O$ does not belong to $C$, it suffices to show that there exists such a point $(O, x, y) \in \Sigma_{C, D_{1}, D_{2}}$ with $E_{1}, E_{2}$ meeting tangentially at $O$ and transversally at the other remaining 7 points. For this, we fix the point $O$ not on $C$, and fix $E_{1}$ (with equation $e_{1}+x c$ ) passing through $O$. We then look at the set of equations $e_{2}+y c$ vanishing at $O$ and tangent to $E_{1}$ at $O$. The restriction of these equations provides a linear system of dimension 2 on $E_{1}$, and one easily checks that for general choice of $C, D_{1}, D_{2}, E_{1}, O$, its base-locus is reduced to the point $O$ with multiplicity 2. By Bertini, the general intersection $E_{1} \cap E_{2}$ for $E_{2}$ as above has only the point $O$ for double point. This proves the claim.

One easily deduces from this that for any choice of $P_{1}, \ldots, P_{7}$, the classes $e_{i}$ of the corresponding exceptional curves of the minimal desingularization $\widetilde{S}$ of $S$ and the class $h=$ $c_{1}\left(\mathcal{O}_{S}(1)\right)$ generate a primitive sublattice of $H^{2}(\widetilde{S}, \mathbb{Z})$ (equivalently, there are no relations with coefficients in $\mathbb{Z} / 2 \mathbb{Z}$ between these classes). Hence for the very general deformation $S_{t}$ as above, its Picard group is freely generated by the classes $e_{i}, i=1, \ldots, 7$, and $h$. Let $\tau: X \rightarrow \mathbb{P}^{3}$ be the blow-up of $\mathbb{P}^{3}$ at the points $P_{1, t}, \ldots, P_{7, t}$, with exceptional divisors $D_{1, t}, \ldots, D_{7, t}$ intersecting the proper transform $\widetilde{S}_{t}$ along $E_{i, t}$. The surface $\widetilde{S}_{t}$ belongs to the linear system $|L|, L:=\tau^{*}\left(\mathcal{O}_{\mathbb{P}^{3}}(4)\right)\left(-2 \sum_{i} D_{i, t}\right)$ and we want to prove that $\operatorname{dim}|L|=6$. As $H^{1}\left(X, \mathcal{O}_{X}\right)=0$, this is equivalent to saying that $h^{0}\left(\widetilde{S}_{t}, L_{\mid \widetilde{S}_{t}}\right)=6$, and also to $h^{1}\left(\widetilde{S}_{t}, L_{\mid \widetilde{S}_{t}}\right)=$ 0 . Let $L_{t}:=L_{\mid \widetilde{S}_{t}}$. As $L_{t}$ is big, this last vanishing is satisfied if $L_{t}$ is numerically effective, hence if the linear system $\left|L_{t}\right|$ has no base curve on which $L_{t}$ has negative degree, which is equivalent (see [20. Chapter 2,1.6]) to saying that there is no smooth rational curve $C \subset \widetilde{S}_{t}$ such that

$$
L_{t} . C<0 \text {, and } C^{2}=-2
$$

with $L_{t}(-C)$ effective. Let $C$ be such a curve, and write its class $c \in H^{2}\left(\widetilde{S}_{t}, \mathbb{Z}\right)$ as

$$
c=\lambda h+\sum_{i} n_{i} e_{i},
$$

with $\lambda, n_{i}$ integers. Furthermore $n_{i} \leq 0$ as otherwise $C$ has to be one of the $E_{i, t}$, and does not satisfy the condition $L_{t} . C<0$. The two numerical conditions (15) write

$$
4 \lambda^{2}-2 \sum_{i=1}^{7} n_{i}^{2}=-2,16 \lambda+4 \sum_{i=1}^{7} n_{i}<0
$$

Of course one has $4>\lambda>0$ because $C$ is effective and $L_{t}(-C)$ is effective. In fact, the case $\lambda=3$ is impossible because the linear system $\left|L_{t}(-C)\right|$ has dimension $\geq 5$. So only $\lambda=1,2$ are possible.

For $\lambda=1$ we get from (16)

$$
2-\sum_{i=1}^{7} n_{i}^{2}=-1,4+\sum_{i=1}^{7} n_{i}<0 .
$$

and for $\lambda=2$ we get

$$
8-\sum_{i=1}^{7} n_{i}^{2}=-1,8+\sum_{i=1}^{7} n_{i}<0 .
$$


It is easy to check that neither (17) nor (18) has an integral solution with all $n_{i}$ 's $\leq 0$. and this concludes the proof of the sublemma.

Sublemma 1.9 tells us that the fibers of $\phi \circ \psi$ are at most 6-dimensional. Hence

$$
\operatorname{dim}(\operatorname{Im} \phi \circ \psi)=21=\operatorname{dim}\left(\mathbb{P}^{3}\right)^{(7)},
$$

which concludes the proof of Lemma 1.7

We conclude this section with the following result which concerns property 4 of Theorem 0.7 and more generally property $(*)$ :

Theorem 1.10. (cf. Theorem[0.9, (ii)) Let $\widetilde{X}$ be the natural desingularization of a general quartic double solid $X$ with 7 nodes. Then $\tilde{X}$ admits no universal codimension 2 cycle $Z \in C H^{2}\left(J^{3}(\widetilde{X}) \times \widetilde{X}\right)$.

More precisely, there is no smooth connected projective variety $B$ equipped with a codimension 2 cycle $Z \in C H^{2}(B \times \widetilde{X})$ which is cohomologous to 0 on fibers $b \times X, b \in B$, and such that the morphism $\Phi_{Z}: B \rightarrow J^{3}(\widetilde{X})$ induced by the Abel-Jacobi map of $\widetilde{X}$ is surjective with rationally connected general fibers.

Proof. We use the following strengthening of Theorem 0.7 (this is 28, Theorem 4.9] combined with the result of [27] guaranteeing the algebraicity of $H^{4}(Y, \mathbb{Z})$ for $Y$ a rationally connected threefold):

Theorem 1.11. Let $Y$ be a rationally connected 3-fold satisfying the following properties:

(a) $H^{*}(Y, \mathbb{Z})$ has no torsion;

(b) There is a codimension 2-cycle $Z \in \mathrm{CH}^{2}(B \times Y)$ inducing a surjective map $\Phi_{Z}$ : $B \rightarrow J^{3}(Y)$ with rationally connected general fibers;

(c) $J^{3}(Y)$ has a 1-cycle in the minimal cohomology class $\theta^{g-1} /(g-1) !, g=\operatorname{dim} J^{3}(Y)$.

Then $Y$ admits an integral cohomological decomposition of the diagonal.

Take now for $Y$ the desingularization $\tilde{X}$ of the general double solid $X$ with 7 nodes. Then property (a) holds as already mentioned. The property $\operatorname{dim} J^{3}(\widetilde{X})=3$ is satisfied for any double solid $X \rightarrow \mathbb{P}^{3}$ ramified along a nodal quartic with 7 nodes imposing independent conditions to quadrics in $\mathbb{P}^{3}$, see [8, Corollary 2.32]. Property (c) is thus satisfied in our case because the set $z$ of nodal points is general, so that $\operatorname{dim} J^{3}(\widetilde{X})=3$, and any ppav of dimension 3 is a Jacobian. As Theorem 1.6 says that $\tilde{X}$ does not admit an integral cohomological decomposition of the diagonal, we conclude that (b) must fail.

\section{Application to the universal degree 3 unramified co- homology with torsion coefficients}

We prove in this section Corollary 0.11 that we will get as a direct consequence of the following result (cf. Theorem 0.10 ):

Theorem 2.1. Let $X$ be a smooth complex projective variety of dimension $n$ with $\mathrm{CH}_{0}(X)=$ $\mathbb{Z}$. Assume

1. $H^{*}(X, \mathbb{Z})$ has no torsion and the Künneth components $\delta_{0}, \ldots, \delta_{4}$ of the diagonal are algebraic.

2. The group $H_{n r}^{3}(X, \mathbb{Q} / \mathbb{Z}$ ) is trivial (or equivalently by [12], the integral Hodge classes of degree 4 on $X$ are algebraic).

Then the degree 3 unramified cohomology of $X$ is universally trivial if and only if there is a universal codimension 2 cycle $Z \in \mathrm{CH}^{2}\left(J^{3}(X) \times X\right)$. 
Here and below, the Künneth components $\delta_{i}$ act as identity on $H^{i}(X, \mathbb{Z})$, and as 0 on $H^{j}(X, \mathbb{Z})$ for $i \neq j$. They are well-defined because $H^{*}(X, \mathbb{Z})$ is torsion-free.

Example 2.2. This theorem applies for example to cubic 4-folds. Indeed, by [30, they satisfy the integral Hodge conjecture in degree 4 and the other assumptions are easy to check. Obviously, they have trivial $\mathrm{CH}_{0}$ group. The fact that there is no torsion in the cohomology of a smooth hypersurface in projective space is a consequence of the Lefschetz theorem on hyperplane sections. Finally, the Künneth components of their diagonal are algebraic, because their cohomology groups of degree 6 and 2 are cyclic, generated by the class $\gamma$ of a line and the class $h$ of a hyperplane section respectively. Thus the components $\delta_{2}$ and $\delta_{6}$ which are the projectors on $H^{2}$ and $H^{6}$ respectively are given by $\delta_{2}=\gamma \otimes h$ and $\delta_{6}=h \otimes \gamma$. It follows that the remaining Künneth component

$$
\delta_{4}=\left[\Delta_{X}\right]-\delta_{0}-\delta_{8}-\delta_{2}-\delta_{6}
$$

is algebraic.

As their intermediate Jacobian is trivial, one concludes by Theorem 2.1 that their third unramified cohomology with torsion coefficients is universally trivial. This generalizes the main result of 2] with a completely different proof. Auel, Colliot-Thélène and Parimala prove that the unramified cohomology of degree 3 with torsion coefficients of a very general cubic fourfold $X$ containing a plane is universally trivial. Their method uses the $K$-theory of quadric bundles.

We get similarly

Corollary 2.3. Let $X$ be a rationally connected threefold with no torsion in $H^{3}(X, \mathbb{Z})$. Then the third unramified cohomology of $X$ with coefficients in $\mathbb{Q} / \mathbb{Z}$ is not universally trivial if and only if $X$ does not admit a universal codimension 2 cycle.

Proof. We just have to check the assumptions of Theorem 2.1. As $X$ is rationally connected, we clearly have $\mathrm{CH}_{0}(X)=\mathbb{Z}$ and furthermore, the fact that $H^{3}(X, \mathbb{Z})$ has no torsion implies that the whole integral cohomology $H^{*}(X, \mathbb{Z})$ has no torsion. The Künneth components $\delta_{2}$ and $\delta_{4}$ of the diagonal of $X$ (which act by projection on $H^{2}(X, \mathbb{Z})$ and $H^{4}(X, \mathbb{Z})$ respectively) are algebraic because $H^{2}(X, \mathbb{Z})$ and $H^{4}(X, \mathbb{Z})$ are generated by algebraic classes. (Note that for the cohomology group $H^{4}(X, \mathbb{Z})$, the fact that it is generated by classes of curves is not obvious and proved in 27].) Thus the last component

$$
\delta_{3}=\left[\Delta_{X}\right]-\delta_{0}-\delta_{2}-\delta_{4}-\delta_{6}
$$

is also algebraic. Finally, assumption 2 in Theorem 2.1 reduces again to the fact already mentioned that $H^{4}(X, \mathbb{Z})$ is algebraic.

Corollary 2.4. (cf. Corollary 0.11 ) Let $X$ be the natural desingularization of a very general quartic double solid with 7 nodes. Then the third unramified cohomology group of $X$ with torsion coefficients is not universally trivial.

Proof. Indeed, there is no torsion in $H^{3}(X, \mathbb{Z})$; this has been already mentioned before and is proved by Endrass [15. As we know by Theorem 1.10 that the desingularization of the very general double solid $X$ with 7 nodes does not admit a universal codimension 2 cycle, the corollary is thus a consequence of Corollary 2.3 ,

Proof of Theorem 2.1. Let us first show that if $X$ has trivial $\mathrm{CH}_{0}$ group, satisfies the assumptions 1 and 2 of the theorem and has no universal codimension 2 cycle, then it has a nontrivial universal third unramified cohomology group with torsion coefficients. We recall from the introduction that the meaning of this statement is that there exist a smooth quasiprojective variety $U$, and an unramified cohomology class $\alpha \in H_{n r}^{3}(U \times X, \mathbb{Q} / \mathbb{Z})$ with the 
property that for any Zariski dense open subset $U^{\prime} \subset U, \alpha_{\mid U^{\prime} \times X}$ is not the pull-back of a cohomology class $\beta \in H^{3}\left(U^{\prime}, \mathbb{Q} / \mathbb{Z}\right)$.

The fact that $H^{*}(X, \mathbb{Z})$ has no torsion implies that there is a Künneth decomposition of cohomology with integral coefficients of $U \times X$ for any variety $U$. On the other hand, the fact that the Künneth components $\delta_{i}, 0 \leq i \leq 4$, of the diagonal of $X$ (which are defined in integral coefficients cohomology) are algebraic implies that for any $U$ and any algebraic cycle $z$ of codimension $\leq 2$ on $U \times X$, the Künneth components of $[z]$ are algebraic, since they are obtained by applying the correspondences $\delta_{i}, 0 \leq i \leq 4$, seen as relative self-correspondences of $U \times X \times X$ over $U$, to $[z]$.

According to [12, where the result is stated for smooth projective varieties but works in the smooth quasi-projective case as well, there is an exact sequence, for any smooth quasi-projective $Y$ :

$$
0 \rightarrow H_{n r}^{3}(Y, \mathbb{Z}) \otimes \mathbb{Q} / \mathbb{Z} \rightarrow H_{n r}^{3}(Y, \mathbb{Q} / \mathbb{Z}) \rightarrow \operatorname{Tors}\left(H^{4}(Y, \mathbb{Z}) / H^{4}(Y, \mathbb{Z})_{\text {alg }}\right) \rightarrow 0,
$$

where $H^{4}(Y, \mathbb{Z})_{\text {alg }} \subset H^{4}(Y, \mathbb{Z})$ is the subgroup of cycle classes $[Z], Z \in \mathrm{CH}^{2}(Y)$. Using this exact sequence, in order to prove that the third unramified cohomology group of $X$ is not universally trivial, it suffices to exhibit a smooth projective variety $B$, and a degree 4 cohomology class $\alpha$ on $B \times X$, such that $N \alpha$ is algebraic for some $N \neq 0$, but $\alpha_{\mid U \times X}$ cannot be written as a sum $a+p r_{1}^{*} b$, with $a$ algebraic on $U \times X$ and $b \in H^{4}(U, \mathbb{Z})_{\text {tors }}$, for any dense Zariski open set $U \subset B$. Such a class indeed provides a torsion class $\bar{\alpha}$ in $H^{4}(B \times X, \mathbb{Z}) / H^{4}(B \times X, \mathbb{Z})_{\text {alg }}$; by the exactness on the right in (19), there exists a lift $\tilde{\bar{\alpha}} \in H_{n r}^{3}(B \times X, \mathbb{Q} / \mathbb{Z})$; then $\tilde{\bar{\alpha}}_{\mid U \times X}$ is not in $p r_{1}^{*} H^{3}(U, \mathbb{Q} / \mathbb{Z})$ for any $U \subset B$ dense Zariski open. Indeed, we have a commutative diagram

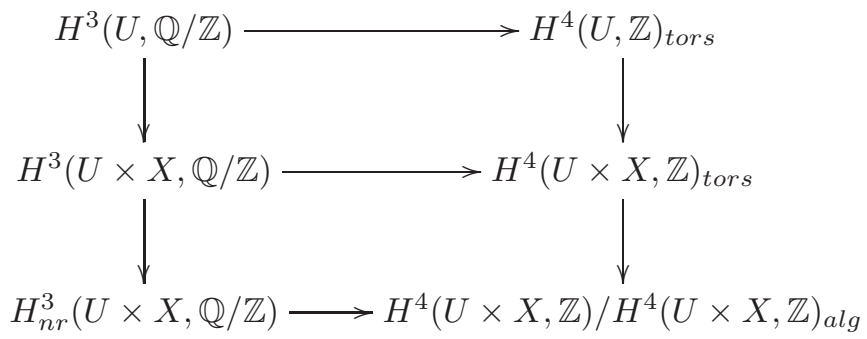

where the first two vertical maps are pull-back maps $p r_{1}^{*}$, and the first two horizontal ones are induced by the exact sequence

$$
0 \rightarrow \mathbb{Z} \rightarrow \mathbb{Q} \rightarrow \mathbb{Q} / \mathbb{Z} \rightarrow 0
$$

The third horizontal map is the last map of (19) for $Y=U \times X$. So if $\tilde{\bar{\alpha}}_{\mid U \times X}$ belonged to $p r_{1}^{*} H^{3}(U, \mathbb{Q} / \mathbb{Z})$, its image in $H^{4}(U \times X, \mathbb{Z}) / H^{4}(U \times X, \mathbb{Z})_{a l g}$, which is also the restriction of $\bar{\alpha}$ to $U \times X)$, would come from a torsion class in $H^{4}(U, \mathbb{Z})$, which we have excluded.

We now construct $B$ and $\alpha$ : We take $B:=J^{3}(X)$. We first prove:

Lemma 2.5. There is an integer $N$, and a codimension 2 cycle $Z_{N} \in \mathrm{CH}^{2}\left(J^{3}(X) \times X\right)$ such that $Z_{N \mid t \times X}$ is cohomologous to 0 for any $t \in J^{3}(X)$ and

$$
\Phi_{Z_{N}}: J^{3}(X) \rightarrow J^{3}(X), t \mapsto A J_{X}\left(Z_{N, t}\right)
$$

is equal to $N \operatorname{Id}_{J^{3}(X)}$.

Proof. Indeed, as we know that $\mathrm{CH}_{0}(X)=\mathbb{Z}$, the Abel-Jacobi map

$$
\Phi_{X}: \mathrm{CH}^{2}(X)_{h o m} \rightarrow J^{3}(X)
$$

is surjective (see [5]). Hence there exist a variety $W$ and a codimension 2 cycle $Z \in \mathrm{CH}^{2}(W \times$ $X$ ) such that

$$
\Phi_{Z}: W \rightarrow J^{3}(X), \Phi_{Z}(t)=A J_{X}\left(Z_{t}\right),
$$


is a surjective morphism of algebraic varieties. Replacing $W$ by a linear section if necessary, we may assume that $\Phi_{Z}: W \rightarrow J^{3}(X)$ is generically finite of degree $N$. We now take for $Z_{N}$ the cycle $\left(\Phi_{Z}, I d_{X}\right) *(Z)$. It is immediate to check that $\Phi_{Z_{N}}: J^{3}(X) \rightarrow J^{3}(X)$ is equal to $N I d_{J^{3}(X)}$. Thus the lemma is proved.

The condition that $\Phi_{Z_{N}}: J^{3}(X) \rightarrow J^{3}(X)$ is equal to $N I d_{J^{3}(X)}$ is equivalent to the fact that the Künneth component of type $(1,3)$ of $\left[Z_{N}\right]$ induces $N$ times the canonical isomorphism between $H_{1}\left(J^{3}(X), \mathbb{Z}\right)$ and $H^{3}(X, \mathbb{Z})$. Furthermore, as explained above, by taking the Künneth component of type $(1,3)$, we may assume $\left[Z_{N}\right]$ is of Künneth type $(1,3)$. Recall now from Remark 0.6 that there is also an integral (Hodge) class $\alpha$ on $J^{3}(X) \times X$ which is of Künneth type $(1,3)$ and induces the canonical isomorphism between $H_{1}\left(J^{3}(X), \mathbb{Z}\right)$ and $H^{3}(X, \mathbb{Z})$. We thus have

$$
\left[Z_{N}\right]=N \alpha \text { in } H^{4}\left(J^{3}(X) \times X, \mathbb{Z}\right) .
$$

Hence we constructed the class $\alpha$ and to finish we just have to prove the following:

Lemma 2.6. For any dense Zariski open set $U \subset J^{3}(X)$, the image of $\alpha$ in

$$
H^{4}(U \times X, \mathbb{Z}) / H^{4}(U \times X, \mathbb{Z})_{\text {alg }}
$$

is nonzero modulo $\mathrm{pr}_{1}^{*} H^{4}(U, \mathbb{Z})_{\text {tors }}$.

Proof. Otherwise $\alpha_{\mid U \times X}=a+p r_{1}^{*} b$, where $a$ is algebraic and $b$ is torsion, for some dense Zariski open set $U \subset J^{3}(X)$. But $\alpha_{\mid U \times X}$ is of Künneth type $(1,3)$ while $p r_{1}^{*} b$ is of Künneth type $(4,0)$. As the Künneth decomposition, which works as well on $U$, preserves algebraic classes, we conclude by projection on the Künneth component of type $(1,3)$ that $\alpha_{\mid U \times X}=a$ is algebraic on $U \times X$. (Note that, alternatively, we can restrict to a smaller Zariski open set, where the torsion class $b$ vanishes.) This means that there is a decomposition

$$
\alpha=\alpha_{1}+\alpha_{2}
$$

in $H^{4}\left(J^{3}(X) \times X, \mathbb{Z}\right)$, where $\alpha_{1}$ is the class of a cycle $Z$ on $J^{3}(X) \times X$ and $\alpha_{2}$ is a cohomology class supported on $D \times X$, where $D=J^{3}(X) \backslash U$. But then the Künneth component of type $(1,3)$ of $\alpha_{2}$ must be 0 . So the Künneth component of type $(1,3)$ of $Z$ is equal to $\alpha$ and $Z$ is a universal codimension 2 cycle on $J^{3}(X) \times X$, contradicting our assumption.

In the other direction, let us prove that if $X$ satisfies the assumptions of Theorem 2.1 and has a universal codimension 2 cycle, then its third unramified cohomology group with torsion coefficients is universally trivial.

Let thus $B$ be a smooth quasi-projective complex variety and let $\bar{B}$ be a smooth projective completion of $B$. Let $\alpha \in H_{n r}^{3}(B \times X, \mathbb{Q} / \mathbb{Z})$. We want to show that up to shrinking $B$ to a Zariski open set $B^{\prime}, \alpha=p r_{1}^{*} \beta$ for some class $\beta \in H^{3}\left(B^{\prime}, \mathbb{Q} / \mathbb{Z}\right)$. Note that $H_{n r}^{3}(B \times X, \mathbb{Q} / \mathbb{Z})$ contains $H_{n r}^{3}(B \times X, \mathbb{Z}) \otimes \mathbb{Q} / \mathbb{Z}$.

Lemma 2.7. If $X$ has trivial $\mathrm{CH}_{0}$ group, the map pr $r_{1}^{*}: H_{n r}^{3}(B, \mathbb{Z}) \rightarrow H_{n r}^{3}(B \times X, \mathbb{Z})$ is an isomorphism.

Proof. Indeed, this map has a left inverse, namely the restriction map $r_{B \times x}$ to $B \times x$ for any point $x \in X(\mathbb{C})$. Furthermore it follows from the Merkurjev-Suslin theorem (or the BlochKato conjecture) that the groups $H_{n r}^{3}(B, \mathbb{Z})$ and $H_{n r}^{3}(B \times X, \mathbb{Z})$ have no torsion. Thus it suffices to show that the map $p r_{1}^{*}: H_{n r}^{3}(B, \mathbb{Q}) \rightarrow H_{n r}^{3}(B \times X, \mathbb{Q})$ is an isomorphism. This is however implied by the Bloch-Srinivas decomposition of the diagonal (11) for some integer $N \neq 0$, which is satisfied by $X$ since $\mathrm{CH}_{0}(X)=\mathbb{Z}$. This gives as well a decomposition over $B:$

$$
N\left(B \times \Delta_{X}\right)=Z_{1}+Z_{2} \text { in } \mathrm{CH}^{n}(B \times X \times X), n=\operatorname{dim} X
$$


where $Z_{2}=N(B \times X \times x)$ for some point $x \in X(\mathbb{C})$ and $Z_{1}$ is supported on $B \times D \times X$, for some proper closed algebraic subset $D \varsubsetneqq X$. As recalled in [12, Appendix], the various cycles $\gamma$ appearing in this equality act on unramified cohomology of $B \times X$ via

$$
\eta \mapsto \gamma^{*} \eta:=\operatorname{pr}_{B, 1 *}\left(p r_{B, 2}^{*} \eta \cdot[\gamma]_{B O}\right),
$$

where the class $[\gamma]_{B O} \in H^{n}\left((B \times X \times X)_{Z a r}, \mathcal{H}^{n}(\mathbb{Z})\right)$ is the Bloch-Ogus cycle class of $\gamma$ (see [4), and $p r_{B, 1}, p r_{B, 2}$ are the two projections from $B \times X \times X$ to $B \times X$. As $B \times \Delta_{X}$ acts as identity on $H_{n r}^{3}(B \times X, \mathbb{Q})$ and $Z_{1}^{*}$ acts as 0 on $H_{n r}^{3}(B \times X, \mathbb{Q})$, one gets that

$$
N I d_{H_{n r}^{3}(B \times X, \mathbb{Q})}=Z_{2}^{*}=N\left(p r_{1}^{*} \circ r_{B \times x}\right),
$$

which proves the result.

From Lemma 2.7. we see that it suffices to show that, up to passing to a dense Zariski open subset of $B$ if necessary, the image of $\alpha$ in the quotient

$$
\frac{H_{n r}^{3}(B \times X, \mathbb{Q} / \mathbb{Z})}{H_{n r}^{3}(B \times X, \mathbb{Z}) \otimes \mathbb{Q} / \mathbb{Z}}
$$

is 0 . By the exact sequence (19), this quotient is isomorphic to the torsion of the group $H^{4}(B \times X, \mathbb{Z}) / H^{4}(B \times X, \mathbb{Z})_{\text {alg }}$. So the result follows from the following:

Lemma 2.8. Let $X$ satisfy the assumptions of Theorem 2.1. Then, if $X$ has a universal codimension 2 cycle, for any $B$ and any degree 4 class $\alpha \in H^{4}(B \times X, \mathbb{Z})$, such that $N \alpha$ is algebraic for some integer $N \neq 0$, there is a dense Zariski open subset $B^{\prime} \subset B$, such that the class $\alpha$ is algebraic in $B^{\prime} \times X$.

Proof. First of all, as the integral cohomology of $X$ is torsion free, there is a Künneth decomposition

$$
\alpha=\sum_{0 \leq i \leq 4} \alpha_{i, 4-i},
$$

where $\alpha_{i, 4-i} \in H^{i}(B, \mathbb{Z}) \otimes H^{4-i}(X, \mathbb{Z})$. Furthermore, this decomposition is obtained by applying to $\alpha$ the Künneth projectors $\delta_{i}, 0 \leq i \leq 4$ of $X$. As we know that the $\delta_{i}$ 's are algebraic for $i \leq 4$, each class $\alpha_{i, 4-i}$ satisfies the property that $N \alpha_{i, 4-i}$ is algebraic.

Consider first the term $\alpha_{4,0} \in p r_{1}^{*} H^{4}(B, \mathbb{Z})$ and write it $\alpha_{4,0}=p r_{1}^{*} \beta, \beta \in H^{4}(B, \mathbb{Z})$. Then $N \beta$ is algebraic on $B$, so there is a dense Zariski open set $B^{\prime} \subset B$ such that $N \alpha=0$ in $H^{4}\left(B^{\prime}, \mathbb{Z}\right)$. The Bloch-Kato conjecture then implies that there is a dense Zariski open set $B^{\prime \prime} \subset B$ such that $\alpha=0$ in $H^{4}\left(B^{\prime \prime}, \mathbb{Z}\right)$ since it implies that the sheaf $\mathcal{H}^{4}(\mathbb{Z})$ on $B_{Z a r}$ has no $\mathbb{Z}$-torsion.

Next consider the class $\alpha_{2,2} \in p^{*} H^{2}(B, \mathbb{Z}) \otimes H^{2}(X, \mathbb{Z})$ and write it

$$
\alpha_{2,2}=\sum_{i} p r_{1}^{*} \beta_{i} \smile p r_{X}^{*} \alpha_{i}, \beta_{i} \in H^{2}(B, \mathbb{Z}),
$$

where $p r_{X}: B \times X \rightarrow X$ is the second projection and the $\alpha_{i}$ 's form a basis of the free abelian group $H^{2}(X, \mathbb{Z})$, which is generated by divisors classes since $\mathrm{CH}_{0}(X)=\mathbb{Z}$. The group $H^{2 n-2}(X, \mathbb{Z})$ admits a Poincaré dual basis $\alpha_{i}^{*}$, with $\left\langle\alpha_{i}, \alpha_{j}^{*}\right\rangle=\delta_{i j}$. The $\mathbb{Q}$-vector $H^{2 n-2}(X, \mathbb{Q})$ is generated by classes of curves, since $H^{2}(X, \mathbb{Q})$ is generated by divisor classes and for any ample line bundle $L$ on $X$, the topological Chern class $l=c_{1, t o p}(L) \in H^{2}(X, \mathbb{Q})$ provides a Lefschetz isomorphism

$$
l^{n-2} \smile: H^{2}(X, \mathbb{Q}) \cong H^{2 n-2}(X, \mathbb{Q}) .
$$

Thus there exists a nonzero integer $N^{\prime}$ such that $N^{\prime} \alpha_{i}^{*}=\left[z_{i}\right]$ in $H^{2 n-2}(X, \mathbb{Z})$ for some 1-cycles $z_{i} \in C H^{n-1}(X)$. It thus follows that

$$
\beta_{i}=p r_{1 *}\left(\alpha_{2,2} \smile p r_{X}^{*} \alpha_{i}^{*}\right), N^{\prime} \beta_{i}=p r_{1 *}\left(\alpha_{2,2} \smile p r_{X}^{*}\left[z_{i}\right]\right) .
$$


As $N \alpha_{2,2}$ is algebraic, so are the classes $N N^{\prime} \beta_{i}$. This implies that the classes $\beta_{i}$ themselves are algebraic, by the same argument as before since they restrict to torsion classes on some Zariski open set $B^{\prime} \subset B$, and this implies that they have to vanish identically on a dense Zariski open set $B^{\prime \prime} \subset B$.

The term $\alpha_{0,4} \in p r_{X}^{*} H^{4}(X, \mathbb{Z}) \cong H^{4}(X, \mathbb{Z})$ satisfies the condition that $N \alpha_{0,4}$ is algebraic. The assumption 2 made on $X$ implies that $\alpha_{0,4}$ itself is algebraic.

As $H^{1}(X, \mathbb{Z})=0$, we are thus left with $\alpha_{1,3} \in H^{1}(B, \mathbb{Z}) \otimes H^{3}(X, \mathbb{Z})$. We have now:

Lemma 2.9. (i) The class $\alpha_{1,3}$ is the restriction to $B \times X$ of a class $\bar{\alpha}_{1,3} \in H^{1}(\bar{B}, \mathbb{Z}) \otimes$ $H^{3}(X, \mathbb{Z})$ which has the property that $N \bar{\alpha}_{1,3}$ is algebraic.

(ii) There is a morphism $\phi: \bar{B} \rightarrow J^{3}(X)$ such that $\left(\phi, I d_{X}\right)^{*} \alpha=\bar{\alpha}_{1,3}$, where $\alpha$ is the integral Hodge class on $J^{3}(X) \times X$ introduced in Remark 0.6 .

Proof. (i) The class $N \alpha_{1,3}$ extends to an integral cohomology class on $\bar{B} \times X$ because it is algebraic. As the Künneth components of the diagonal of $X$ are algebraic, one may even assume that the class $N \alpha_{1,3}$ extends to an integral cohomology class $\beta$ on $\bar{B} \times X$ which is algebraic and of Künneth type $(1,3)$. As $H^{*}(X, \mathbb{Z})$ has no torsion, this class $\beta$ can be seen as a morphism

$$
\beta_{*}: H_{3}(X, \mathbb{Z}) \rightarrow H^{1}(\bar{B}, \mathbb{Z})
$$

which has the property that, denoting by $r_{B}: H^{1}(\bar{B}, \mathbb{Z}) \rightarrow H^{1}(B, \mathbb{Z})$ the restriction to $B$, the composite morphism

$$
r_{B} \circ \beta_{*}=N \alpha_{1,3_{*}}: H_{3}(X, \mathbb{Z}) \rightarrow H^{1}(B, \mathbb{Z})
$$

is divisible by $N$. On the other hand, it is quite easy to prove that the restriction map $r_{B}$ is injective and that its cokernel is torsion free. Thus the morphism $\beta_{*}$ is also (uniquely) divisible by $N$, and so is $\beta$, which proves (i).

(ii) The class $N \bar{\alpha}_{1,3}$ being algebraic, it is an integral Hodge class on $\bar{B} \times X$, so $\bar{\alpha}_{1,3}$ is also an integral Hodge class. But the morphisms from $\bar{B}$ to $J^{3}(X)$ identify (modulo the translations of $\left.J^{3}(X)\right)$ to the morphisms of complex tori between Alb $\bar{B}$ and $J^{3}(X)$ which themselves identify to the morphisms of Hodge structures

$$
H_{1}(\bar{B}, \mathbb{Z}) / \text { torsion } \rightarrow H^{3}(X, \mathbb{Z})
$$

because $H^{3}(X, \mathbb{Z})$ has no torsion, and finally these morphisms of Hodge structures identify to the integral Hodge classes of Künneth type $(1,3)$ on $\bar{B} \times X$.

Hence the class $\bar{\alpha}_{1,3}$ provides us with a morphism

$$
\phi: \bar{B} \rightarrow J^{3}(X)
$$

and it is a formal fact to prove following the chain of identifications above that

$$
\left(\phi, I d_{X}\right)^{*}(\alpha)=\bar{\alpha}_{1,3} .
$$

The proof is now finished because we assumed that $X$ admits a universal codimension 2 cycle. This is equivalent to saying that the class $\alpha$ of Remark 0.6 is algebraic because $H^{3}(X, \mathbb{Z})$ has no torsion. Lemma 2.9 thus implies that $\alpha_{1,3}$ is algebraic.

The proof of Theorem 2.1 is finished. 


\section{References}

[1] M. Artin, D. Mumford. Some elementary examples of unirational varieties which are not rational, Proc. London Math. Soc. (3) 25 (1972), 75-95.

[2] A. Auel, J.-L. Colliot-Thélène, R. Parimala. Universal unramified cohomology of cubic fourfolds containing a plane, preprint arXiv:1310.6705.

[3] A. Beauville. Variétés rationnelles et unirationnelles, in Algebraic geometry-open problems (Ravello, 1982), 16-33, Lecture Notes in Math., 997, Springer, Berlin, (1983).

[4] S. Bloch, A. Ogus. Gersten's conjecture and the homology of schemes, Ann. Sci. Éc. Norm. Supér., Sér. 4, 7, 181-201 (1974).

[5] S. Bloch, V. Srinivas. Remarks on correspondences and algebraic cycles, Amer. J. of Math. 105 (1983) 1235-1253.

[6] A-M. Castravet. Rational families of vector bundles on curves, International Journal of Mathematics, Vol. 15, No. 1 (2004) 13-45.

[7] I. Cheltsov. Personal communication.

[8] H. Clemens. Double solids, Adv. in Math. 47 (1983), no. 2, 107-230.

[9] H. Clemens, Ph. Griffiths. The intermediate Jacobian of the cubic threefold, Annals of Mathematics. Second Series 95 (2): 281-356 (1972).

[10] J.-L. Colliot-Thélène, M. Ojanguren, Variétés unirationnelles non rationnelles: au-delà de l'exemple d'Artin et Mumford, Invent. math. 97 (1989), no. 1, 141-158.

[11] J.-L. Colliot-Thélène, A. Pirutka. Hypersurfaces quartiques de dimension 3 : non rationalité stable, arXiv:1402.4153.

[12] J.-L. Colliot-Thélène, C. Voisin, Cohomologie non ramifiée et conjecture de Hodge entière, Duke Math. Journal, Volume 161, Number 5, 735-801 (2012).

[13] T. de Fernex, D. Fusi. Rationality in families of threefolds. Rend. Circ. Mat. Palermo (2) 62 (2013), no. 1, 127-135.

[14] A.-J. de Jong, J. Starr. Cubic fourfolds and spaces of rational curves. Illinois J. Math. 48 (2004), no. 2, 415-450.

[15] S. Endrass. On the divisor class group of double solids, Manuscripta Math. 99, 341-358 (1999).

[16] W. Fulton. Intersection Theory. Ergebnisse der Mathematik und ihrer Grenzgebiete 3. Folge, Band 2. Springer (1984).

[17] A. Grothendieck, Hodge's general conjecture is false for trivial reasons, Topology 8 (1969) 299-303.

[18] J. Harris. Galois groups of enumerative problems. Duke Math. J. 46, no. 4, 685-724 (1979).

[19] J. Harris, M. Roth and J. Starr. Curves of small degree on cubic threefolds, Rocky Mountain J. Math. 35 (2005), no. 3, 761-817.

[20] D. Huybrechts. Lectures on K3 surfaces, notes available at http://www.math.uni-bonn.de/people/huybrech/K3Global.pdf

[21] D. Markushevich and A. Tikhomirov, The Abel-Jacobi map of a moduli component of vector bundles on the cubic threefold, J. Algebraic Geometry 10 (2001), 37-62. 
[22] T. Matsusaka. On a characterization of a Jacobian variety. Memo. Coll. Sci. Univ. Kyoto. Ser. A. Math. 32, 1-19 (1959).

[23] J. P. Murre. Applications of algebraic K-theory to the theory of algebraic cycles, in Proc. Conf. Algebraic Geometry, Sitjes 1983, LNM 1124 (1985), 216-261, Springer-Verlag.

[24] E. Peyre. Unramified cohomology of degree 3 and Noether's problem, Invent. math. 171 (2008), no. 1, 191-225.

[25] A. Roitman. The torsion of the group of 0-cycles modulo rational equivalence. Ann. of Math. (2) 111 (1980), no. 3, 553-569.

[26] V. Voevodsky. Motivic cohomology with $\mathbb{Z} /$ l-coefficients, Ann. of Math. (2) 174 (2011), no. $1,401-438$.

[27] C. Voisin. On integral Hodge classes on uniruled and Calabi-Yau threefolds, in Moduli Spaces and Arithmetic Geometry, Advanced Studies in Pure Mathematics 45, 2006, pp. 43-73.

[28] C. Voisin. Abel-Jacobi map, integral Hodge classes and decomposition of the diagonal, J. Algebraic Geom. 22 (2013), no. 1, 141-174.

[29] C. Voisin. On the universal $\mathrm{CH}_{0}$ group of cubic hypersurfaces, preprint arXiv:1407.7261.

[30] C. Voisin. Some aspects of the Hodge conjecture, Jpn. J. Math. 2 (2007), no. 2, 261-296.

[31] C. Voisin. Sur la jacobienne intermédiaire du double solide d'indice deux, Duke Math. J. 57 (1988), no. 2, 629-646.

[32] Z. Xu. A remark on the Abel-Jacobi morphism for the cubic threefold. C. R. Math. Acad. Sci. Paris 351 (2013), no. 1-2, 63-67.

[33] Géométrie des surfaces K3 : modules et périodes, Astérisque 126, Société Mathématique de France (1985).

Centre de mathématiques Laurent Schwartz

91128 Palaiseau Cédex, France

voisin@math.jussieu.fr 